\title{
Transcriptional Regulation of the Peripheral Pathway for the Anaerobic Catabolism of Toluene and $\boldsymbol{m}$-Xylene in Azoarcus sp. CIB
}

\author{
Blas Blázquez ${ }^{\dagger}$ Manuel Carmona and Eduardo Díaz* \\ Department of Microbial and Plant Biotechnology, Centro de Investigaciones Biológicas-Consejo Superior de \\ Investigaciones Científicas, Madrid, Spain
}

OPEN ACCESS

Edited by:

Kian Mau Goh,

Universiti Teknologi Malaysia,

Malaysia

Reviewed by:

Maximino Manzanera,

University of Granada, Spain

Victor Ladero,

Instituto de Productos Lácteos

de Asturias (CSIC), Spain

*Correspondence:

Eduardo Díaz

ediaz@cib.csic.es

${ }^{\dagger}$ Present address:

Blas Blázquez,

Systems Biology Programme, Centro

Nacional de Biotecnología-Consejo

Superior de Investigaciones

Científicas, Madrid, Spain

Specialty section:

This article was submitted to Microbiotechnology, Ecotoxicology

and Bioremediation,

a section of the journal

Frontiers in Microbiology

Received: 08 January 2018

Accepted: 06 March 2018

Published: 22 March 2018

Citation:

Blázquez B, Carmona M and Díaz E

(2018) Transcriptional Regulation

of the Peripheral Pathway

for the Anaerobic Catabolism

of Toluene and m-Xylene in Azoarcus

sp. CIB. Front. Microbiol. 9:506.

doi: 10.3389/fmicb.2018.00506
Alkylbenzenes, such as toluene and m-xylene, are an important class of contaminant hydrocarbons that are widespread and tend to accumulate in subsurface anoxic environments. The peripheral pathway for the anaerobic oxidation of toluene in bacteria consists of an initial activation catalyzed by a benzylsuccinate synthase (encoded by bss genes), and a subsequent modified $\beta$-oxidation of benzylsuccinate to benzoylCoA and succinyl-CoA (encoded by bbs genes). We have shown here that the bss and bbs genes, which are located within an integrative and conjugative element, are essential for anaerobic degradation of toluene but also for $m$-xylene oxidation in the denitrifying beta-proteobacterium Azoarcus sp. CIB. New insights into the transcriptional organization and regulation of a complete gene cluster for anaerobic catabolism of toluene/m-xylene in a single bacterial strain are presented. The bss and bbs genes are transcriptionally coupled into two large convergent catabolic operons driven by the PbssD and PbbsA promoters, respectively, whose expression is inducible when cells grow anaerobically in toluene or $m$-xylene. An adjacent tdiSR operon driven by the PtdiS promoter encodes a putative two-component regulatory system. TdiR behaves as a transcriptional activator of the PbssD, PbbsA, and PtdiS promoters, being benzylsuccinate/(3-methyl)benzylsuccinate, rather than toluene/m-xylene, the inducers that may trigger the TdiS-mediated activation of TdiR. In addition to the TdiSR-based specific control, the expression of the bss and bbs genes in Azoarcus sp. CIB is under an overimposed regulation that depends on certain environmental factors, such as the presence/absence of oxygen or the availability of preferred carbon sources (catabolite repression). This work paves the way for future strategies toward the reliable assessment of microbial activity in toluene/m-xylene contaminated environments.

Keywords: toluene, $m$-xylene, Azoarcus, anaerobic degradation, tdiSR, catabolite repression, biomarker

\section{INTRODUCTION}

Aromatic compounds such as alkylbenzenes, e.g., toluene, xylenes, ethylbenzene and benzene, are an important class of contaminants that are prominently placed amongst the US Agency for Toxic Substances and Disease Registry's list of priority pollutants because of their carcinogenic and/or neurotoxic effects to humans. Since alkylbenzenes are relatively soluble and mobile in water, they are widespread and tend to accumulate in hostile environments, e.g., subsurface 
anoxic environments, where they are of major concern to groundwater quality and ecosystem health. Therefore, the anaerobic degradation of alkylbenzenes is an important practical aspect of bioremediation and becomes crucial for the biogeochemical cycles (Kummel et al., 2013; Rabus et al., 2016; Lueders, 2017). Despite the aerobic degradation of alkylbenzenes has been extensively studied, the microbial catabolism of these aromatic hydrocarbons under anaerobic conditions is much less known, and novel pathways and enzymes have been recently described, some of which are of potential biotechnological interest (Rabus et al., 2016; Lueders, 2017). Toluene has been used widely as a model compound for studying anaerobic alkylbenzene degradation. Toluene can be degraded coupled to anaerobic respiration with nitrate, sulfate, iron (III), manganese (IV) or carbonate serving as terminal electron acceptors (Foght, 2008; Carmona et al., 2009; Rabus et al., 2016). Geobacter metallireducens GS-15 was the first pure bacterial culture described to be able to degrade toluene in anaerobic conditions (Lovley et al., 1989). Several isolates capable of anaerobic toluene degradation have been described since then, including both facultative anaerobes, e.g., beta-proteobacteria of the Azoarcus, "Aromatoleum," Thauera, Georgfuchsia, Herminiimonas genera, and alpha-proteobacteria of the Magnetospirillum genus, and obligate anaerobes, e.g., delta-proteobacteria of the Geobacter, Desulfobacula and Desulfobacterium genera, and some clostridial strains (Wöhlbrand et al., 2013; Bozinovski et al., 2014; Kim et al., 2014; Strijkstra et al., 2014; Rabus et al., 2016; Lueders, 2017; Meyer-Cifuentes et al., 2017). Most of the studies on anaerobic toluene degradation have been made on the denitrifying bacteria Thauera aromatica $\mathrm{K} 172$ and $\mathrm{T} 1$ strains, Azoarcus sp. strain T and "Aromatoleum aromaticum" EbN1 strain (Coschigano and Young, 1997; Leuthner et al., 1998; Krieger et al., 1999; Achong et al., 2001; Hermuth et al., 2002; Leutwein and Heider, 2002; Chakraborty and Coates, 2004; Coschigano and Bishop, 2004; Kube et al., 2004; Kühner et al., 2005).

The first step in the anaerobic catabolism of toluene is the addition of the methyl group of toluene to fumarate to form $(R)$ benzylsuccinate by the strictly anoxic benzylsuccinate synthase (BSS), a glycyl radical enzyme (Figure 1A). The genes $b s s A, b s s B$ and $b s s C$ code for the $\alpha$ (BssA), $\beta$ (BssB) and $\gamma$ (BssC) subunits of the heterohexameric $(\alpha \beta \gamma)_{2}$ BSS (Leuthner et al., 1998; Krieger et al., 2001; Bhandare et al., 2006; Funk et al., 2014; Heider et al., 2016). Conversion to the active, radical-containing form of BSS depends on an activating enzyme (BssD) that belongs to the family of $S$-adenosyl-methionine radical enzymes and that is encoded by a gene (bssD) which is closely associated with the $b s s A B C$ genes constituting the $b s s D C A B$ operon of toluenedegrading bacteria (Figure 2) (Selmer et al., 2005; Heider et al., 2016). The bss operons contain two additional conserved genes, $b s s E$ and $b s s F$ (Figure 2), whose functions are still unknown. Whereas BssE is an essential protein for toluene degradation (Bhandare et al., 2006), the role of BssF in the anaerobic oxidation of toluene has not yet been explored. Moreover, in beta-proteobacteria the bss cluster contains a set of additional genes, i.e., the bssGIJKL genes (Figure 2), whose function is so far unknown. Despite the primary structure of the gene products is conserved in the bss clusters from different denitrifying bacteria, the transcriptional organization of the bss clusters differs between species and even between strains of the same species (Coschigano, 2000; Achong et al., 2001; Kube et al., 2004).

Further anaerobic degradation of toluene consists of a modified beta-oxidation of $(R)$-benzylsuccinate to benzoyl-CoA and succinyl-CoA (Figure 1A). All enzymes of this pathway are encoded by the bbs gene cluster (Figure 2) (Leuthner and Heider, 2000; Leutwein and Heider, 2001, 2002; Kube et al., 2004). Like in the $b s s$ gene cluster, the $b b s$ cluster contains genes, e.g., bbsI and bbsJ, of unknown function (Carmona et al., 2009). Although the gene organization of the bbsABCDEFJGH cluster is usually conserved except in some delta-proteobacteria (Figure 2) (Kube et al., 2004; Butler et al., 2007; Chaurasia et al., 2015), the transcriptional organization of a $b b s$ cluster has not been studied so far in any bacteria.

Upstream of the bss cluster in most denitrifying bacteria there are two genes, $t d i S$ and $t d i R$ (toluene-degradation inducer) (Figure 2) that were proposed to encode the sensor histidine kinase and the cognate response regulator, respectively, of a two-component regulatory system likely involved in the transcriptional control of the catabolic bss and bbs genes (Coschigano, 2000; Achong et al., 2001; Hermuth et al., 2002; Kube et al., 2004; Kühner et al., 2005). Extracts of Escherichia coli cells that expressed the $t d i R$ gene from $T$. aromatica $\mathrm{K} 172$ were able to retard the migration of a DNA probe that contained the $b s s D$ promoter, supporting the notion that TdiR likely behaves as a transcriptional regulator of the bss genes (Leuthner and Heider, 1998). Nevertheless, a clear experimental demonstration that TdiS and TdiR control the expression of both bss and bbs genes in beta-proteobacteria is still missing.

Unlike toluene degradation, anaerobic degradation of xylenes (meta-, ortho-, and para-xylene) has been much less studied. The first reaction of the $m$-xylene degradation pathway is performed by the BSS enzyme that catalyzes the addition of fumarate to the methyl group to form (3-methyl)benzylsuccinate (Figure 1A) (Krieger et al., 1999; Verfürth et al., 2004). The (3methyl)benzylsuccinate was also detected as a key intermediate of the fumarate addition pathway in xylene-degrading sulfatereducing cultures (Morasch et al., 2004; Herrmann et al., 2009). On the other hand, it has been proposed that the bbs gene products might be responsible of the $\beta$-oxidation of (3methyl)benzylsuccinate to 3-methylbenzoyl-CoA and succinylCoA in a similar way than in the catabolism of toluene (Figure 1A) (Krieger et al., 1999; Bozinovski et al., 2014), although there is not yet a genetic demonstration that $b b s$ genes are responsible for anaerobic $m$-xylene degradation.

Azoarcus sp. CIB is facultative anaerobic bacterium able to grow aerobically in toluene and anaerobically (nitrate-reducing) in toluene and $m$-xylene (López-Barragán et al., 2004). Moreover, Azoarcus sp. CIB also shows an endophitic lifestyle (Fernández et al., 2014) and is able to resist some metals and metalloids (Fernández-Llamosas et al., 2016). The strain CIB has been used previously to decipher the transcriptional organization and regulation of several gene clusters involved in the anaerobic degradation of aromatic compounds and in the resistance strategies to survive in the presence of high concentrations of these contaminants (López-Barragán et al., 2004; Blázquez et al., 


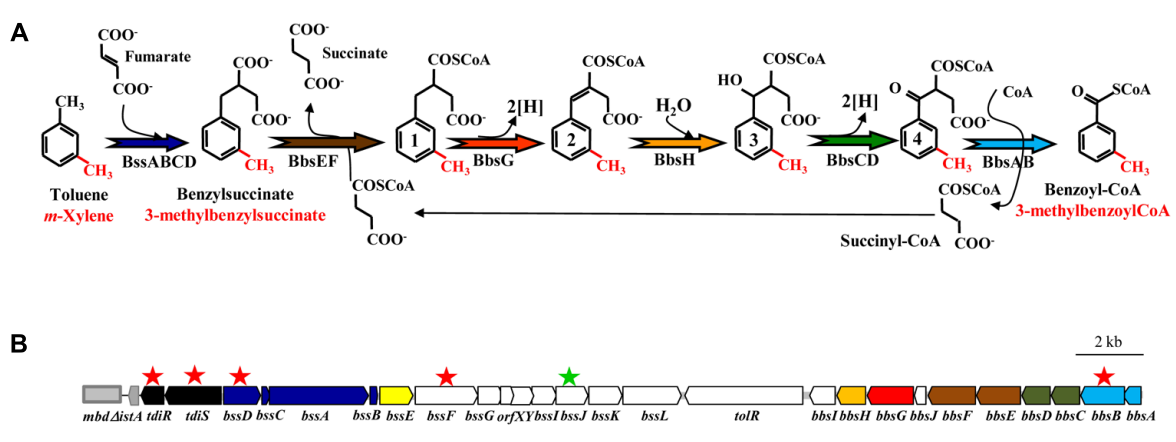

FIGURE 1 | Proposed toluene and $m$-xylene anaerobic peripheral degradation pathway in Azoarcus sp. CIB: (A) Scheme of the proposed peripheral pathway of anaerobic degradation of toluene and $m$-xylene. The enzymes are indicated following the color code indicated in (B). Toluene and $m$-xylene derivatives are shown in black and red color, respectively. Intermediates 1 correspond to benzylsuccinyl-CoA (black) and (3-methyl)benzylsuccinyl-CoA (red). Intermediates 2 correspond to phenylitaconyl-CoA (black) and (3-methyl)phenylitaconyl-CoA (red). Intermediates 3 correspond to 2-[hydroxyphenyl]-succinyl-CoA (black) and 2-[hydroxyphenyl(methyl)]-succinyl-CoA (red). Intermediates 4 correspond to benzoylsuccinyl-CoA (black) and (3-methyl)benzoylsuccinyl-CoA (red). Modified from Carmona et al. (2009). (B) Scheme of the gene cluster encoding the anaerobic peripheral pathway of toluene and $m$-xylene in Azoarcus sp. CIB. Genes are represented by arrows and their predicted function is annotated as follows: black, regulatory genes; dark blue, genes encoding the (3-methyl)benzylsuccinate synthase (BSS); yellow, gene encoding a putative BSS chaperone; orange, gene encoding the (3-methyl)phenylitaconyl-CoA hydratase; red, gene encoding the (3-methyl)benzylsuccinyl-CoA dehydrogenase; brown, genes encoding the succinyl-CoA:(3-methyl)benzylsuccinate CoA transferase; green, genes encoding a 2-[hydroxyphenyl(methyl)]-succinyl-CoA dehydrogenase; light blue, genes encoding the (3-methyl)benzoylsuccinyl-CoA thiolase; white, genes of unknown function. A truncated IS21 transposase ist $A$ gene $(\Delta i s t A)$ is shown by a gray arrow. The gray rectangle indicates the $m b d$ cluster responsible for the $3-$ methylbenzoyl-CoA central pathway. Genes that have been inactivated in this work and avoid the use of toluene and $m$-xylene as sole carbon and energy source by the corresponding mutant strains (Azoarcus sp. ClBdtidR, Azoarcus sp. ClBdtidS, Azoarcus sp. ClBdbssD, Azoarcus sp. ClBdbssF, Azoarcus sp. ClBdbbsB) are indicated with a red star. The gene that has been inactivated and does not avoid the use of toluene and $m$-xylene as sole carbon and energy source in the corresponding mutant strain (Azoarcus sp. ClBdbssJ) is indicated with a green star.

2008; Carmona et al., 2009; Valderrama et al., 2012, 2014; Juárez et al., 2013; Juárez et al., 2015; Martín-Moldes et al., 2016; Zamarro et al., 2016). An in silico analysis of the Azoarcus sp. CIB genome sequence revealed the presence of a chromosomal region within the integrative and conjugative ICEXTD element coding for proteins with significant amino acid sequence identity with Bss and Bbs proteins in closely related bacteria from the Azoarcus, "Aromatoleum" and Thauera genera (Martín-Moldes et al., 2015; Zamarro et al., 2016). This sequence comparison analysis allowed us to propose a similar biochemical pathway for the anaerobic conversion of toluene and $m$-xylene to benzoyl-CoA and 3-methylbenzoyl-CoA, respectively, in strain CIB (Figure 1). Further degradation of benzoyl-CoA and 3-methylbenzoyl-CoA in Azoarcus sp. CIB proceeds via the central bzd (stands for benzoate degradation) and mbd (stands for methylbenzoate degradation) pathways, respectively (López-Barragán et al., 2004; Juárez et al., 2013). In this work we have used Azoarcus sp. CIB to study for the first time the transcriptional organization and regulation of the complete $b s s-b b s$ cluster in a facultative anaerobe. Our results reveal that both the bss and $b b s$ genes are essential for the anaerobic degradation of toluene and $m$-xylene.

\section{MATERIALS AND METHODS}

\section{Bacterial Strains, Plasmids and Growth Conditions}

The E. coli and Azoarcus strains, as well as the plasmids used in this study, are detailed in Table 1. E. coli strains were grown at $37^{\circ} \mathrm{C}$ in Lysogeny Broth (LB) mediun (Bertani, 1951). Azoarcus sp. strain CIB and its derivatives were grown anaerobically under nitrate reducing conditions $\left(10 \mathrm{mM}\right.$ nitrate) at $30^{\circ} \mathrm{C}$ in $\mathrm{MC}$ medium as previously described (López-Barragán et al., 2004). Aromatic hydrocarbons such as toluene, xylenes, styrene were supplied at $250 \mathrm{mM}$ in an inert carrier phase of 2,2,4,4,6,8,8heptamethylnonan (HMN). Benzylsuccinate was added at $4 \mathrm{mM}$ to the culture medium as inducer. Organic acids such as succinate or pyruvate were added at $0.2 \%(\mathrm{w} / \mathrm{v})$. Azoarcus sp. CIB cells were also cultivated aerobically in MC medium in the absence of nitrate. When using toluene aerobically as sole carbon source, it was added directly to the culture medium at $1 \mathrm{mM}$. Where appropriate, antibiotics were added to the culture medium at the following concentrations: ampicillin, $100 \mu \mathrm{g} / \mathrm{ml}$; gentamicin, $10 \mu \mathrm{g} / \mathrm{ml}$; kanamycin, $50 \mu \mathrm{g} / \mathrm{ml}$. Growth was determined by measuring the optical density at $600 \mathrm{~nm}\left(\mathrm{OD}_{600}\right)$ in a Shimadzu UV-260 spectrophotometer.

\section{Molecular Biology Techniques}

Standard molecular biology techniques were performed as previously described (Sambrook and Russell, 2001). DNA fragments were purified with Gene-Clean Turbo (BIO101 Systems); plasmids and PCR products were purified with a High Pure Plasmid and PCR Product Purifications kits (Roche), respectively. Oligonucleotides were supplied by Sigma Co. All cloned inserts and DNA fragments were confirmed by DNA sequencing with fluorescently labeled dideoxynucleotide terminators (Sanger et al., 1977) and AmpliTaq FS DNA polymerase (Applied Biosystems) in an ABI Prism 377 automated DNA sequencer (Applied Biosystems). Transformation of E. coli cells was carried out by using the $\mathrm{RbCl}$ method or by 


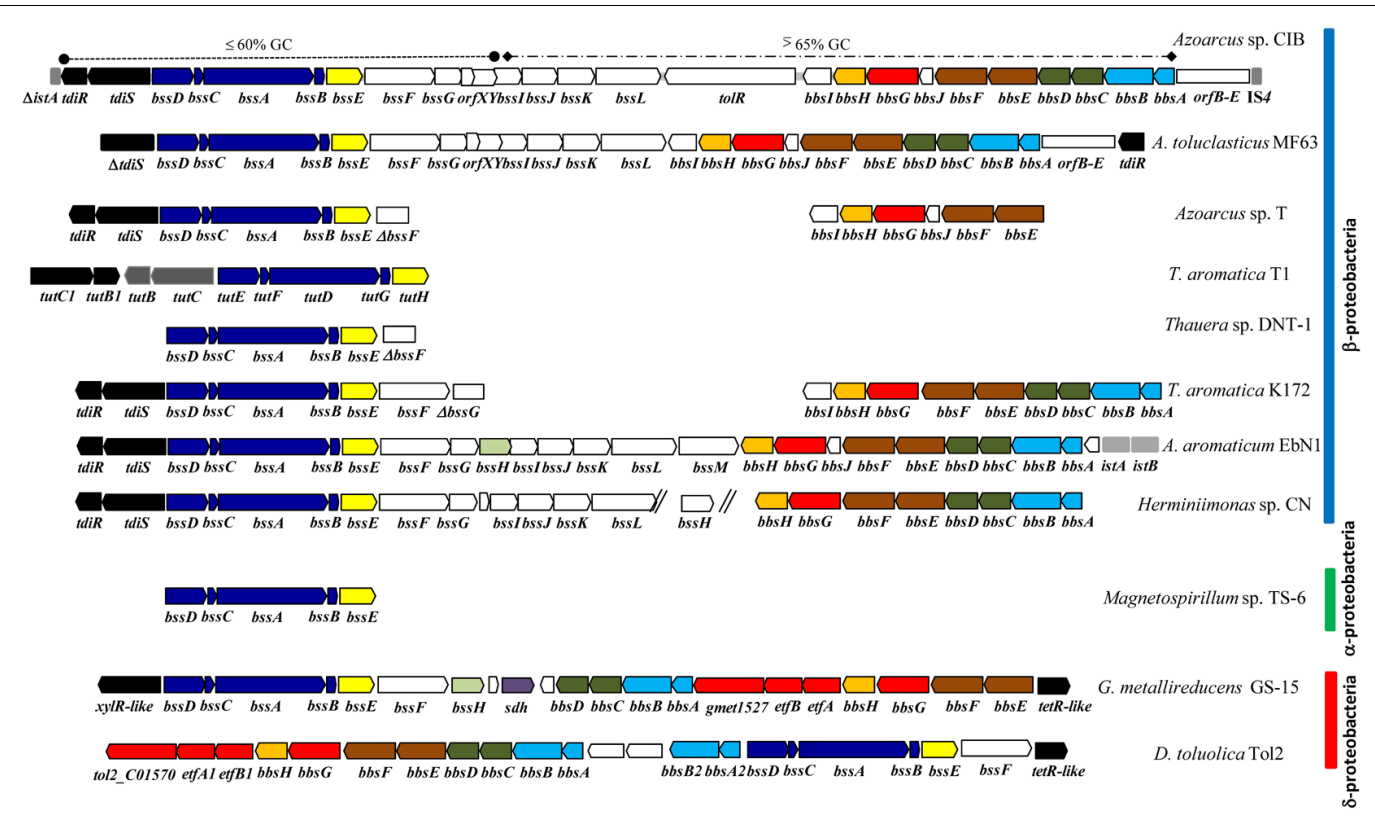

FIGURE 2 | Scheme of the genetic organization of the tdi-bss-bbs cluster in different bacteria. The tdi-bss-bss clusters from Azoarcus sp. CIB, Azoarcus toluclasticus MF63 (Acc. No. NZ_ARJX00000000.1), Azoarcus sp. strain T (Ac. No. AY032676), T. aromatica T1 (Ac. No. U57900 and AF113168), Thauera sp. strain DNT-1 (Ac. No. AB066263), T. aromatica K172 (Ac. No. AJ001848 and AF173961), "Aromatoleum aromaticum" EbN1 (Ac. No. NC_006513), Herminiimonas sp. CN (Ac. No. AVCC01000000), Magnetospirillum sp. strain TS-6 (Ac. No. AB167725), Geobacter metallireducens GS-15 (Ac. No. NC_007517) and Desulfobacula toluolica Tol2 (Ac. No. NC_018645) are represented here. Genes are represented by arrows following the color code indicated in Figure 1: black, regulatory genes; gray, putative regulatory genes of an aerobic toluene degradation pathway; dark blue, genes encoding the (3-methyl)benzylsuccinate synthase (BSS); yellow, genes encoding a putative BSS chaperone; orange, genes encoding the (3-methyl)phenylitaconyl-CoA hydratase; red, genes encoding the (3-methyl)benzylsuccinyl-CoA dehydrogenase and its predicted associated electron-transfer system; brown, genes encoding the succinyl-CoA:(3-methyl)benzylsuccinate CoA transferase; green, genes encoding a 2-[hydroxyphenyl(methyl)]-succinyl-CoA dehydrogenase; light blue, genes encoding the (3-methyl)benzoylsuccinyl-CoA thiolase; light green, genes encoding a putative toluene transport system; violet, gene encoding a putative succinate-dehydrogenase flavoprotein; white, genes of unknown function. Insertion sequences are indicated by gray rectangles. The white rectangle next to the bbs operon in Azoarcus sp. CIB represents orfB (AzClB_4527), orfC (AzClB_4528), orfD (AzCIB_4529) and orfE (AzCIB_4530) genes, that are also present in A. toluclasticus MF63. In the bss-bbs cluster of Azoarcus sp. CIB, the DNA fragment harboring the bssDCABEFG genes and whose average GC content is $58 \%$ is indicated by a disontinuous line flanked by circles. The DNA fragment containing the rest of the bss (bss/JKL) and bbs genes, whose average GC content is $65 \%$, is indicated by a discontinuous line flanked by diamonds.

electroporation (Gene Pulser, Bio-Rad) (Sambrook and Russell, 2001). Plasmids were transferred from E. coli S17-1 $\lambda$ pir (donor strain) into Azoarcus sp. recipient strains by biparental filter mating as described previously (López-Barragán et al., 2004).

\section{Construction of Azoarcus sp. ClBdtdiR, Azoarcus sp. ClBdtdiS, Azoarcus sp. CIBdbssD, Azoarcus sp. CIBdbssF, Azoarcus sp. ClBdbssJ, and Azoarcus sp. ClBdbbsB Strains}

For insertional disruption of the genes through single homologous recombination, an internal region of each gene was PCR-amplified, cloned into plasmid pGEM-T Easy, and subcloned into the pK18mob suicide vector (Table 1). For gene disruption of the $t d i R$ gene, a 0.4 -kb internal fragment was PCRamplified using primers tdiRint5 and tdiR131.5 (Supplementary Table S1), and cloned in plasmid pGEM-tdiR (Table 1). The pGEM-tdiR plasmid was XbaI/PstI double-digested and the 0.4 -kb fragment was then subcloned giving rise to the suicide plasmid pK18mobtdiR (Table 1). For gene disruption of the
tdiS gene, a 0.6-kb internal fragment was PCR amplified using primers 5TdiS and 3TdiS (Supplementary Table S1), and cloned in plasmid pGEM-tdiS (Table 1). The pGEM-tdiS plasmid was EcoRI digested and the 623-bp fragment was then subcloned producing plasmid pK18mobtdiS (Table 1). The tdiS mutant allows expression of the downstream $t d i R$ gene by transcription from the promoter of the kanamycin resistance gene present in the suicide plasmid. For gene disruption of the $b s s D$ gene, a 0.6-kb internal fragment was PCR amplified using primers 5BssD and 3BssD (Supplementary Table S1), and cloned in plasmid pGEM-bssD (Table 1). The pGEM-bssD plasmid was PstI/BamHI double-digested and the 593-bp fragment was then subcloned into pK18mob producing plasmid pK18mobbssD (Table 1). For gene disruption of the $b s s F$ gene, a $0.4-\mathrm{kb}$ internal fragment was PCR-amplified using primers $5 \mathrm{BssF}$ and $3 \mathrm{BssF}$ (Supplementary Table S1) and cloned in plasmid pGEM-bssF (Table 1). The pGEM-bssF plasmid was HindIII/XbaI doubledigested and the 451-bp fragment was then subcloned giving rise to the suicide plasmid pK18mobbssF (Table 1). For gene disruption of the bss gene, a $0.3-\mathrm{kb}$ internal fragment was PCR amplified using primers 5BssJ and 3BssJ (Supplementary Table S1) and cloned in plasmid pGEM-bssJ (Table 1). The 
TABLE 1 | Bacterial strains and plasmids used in this work.

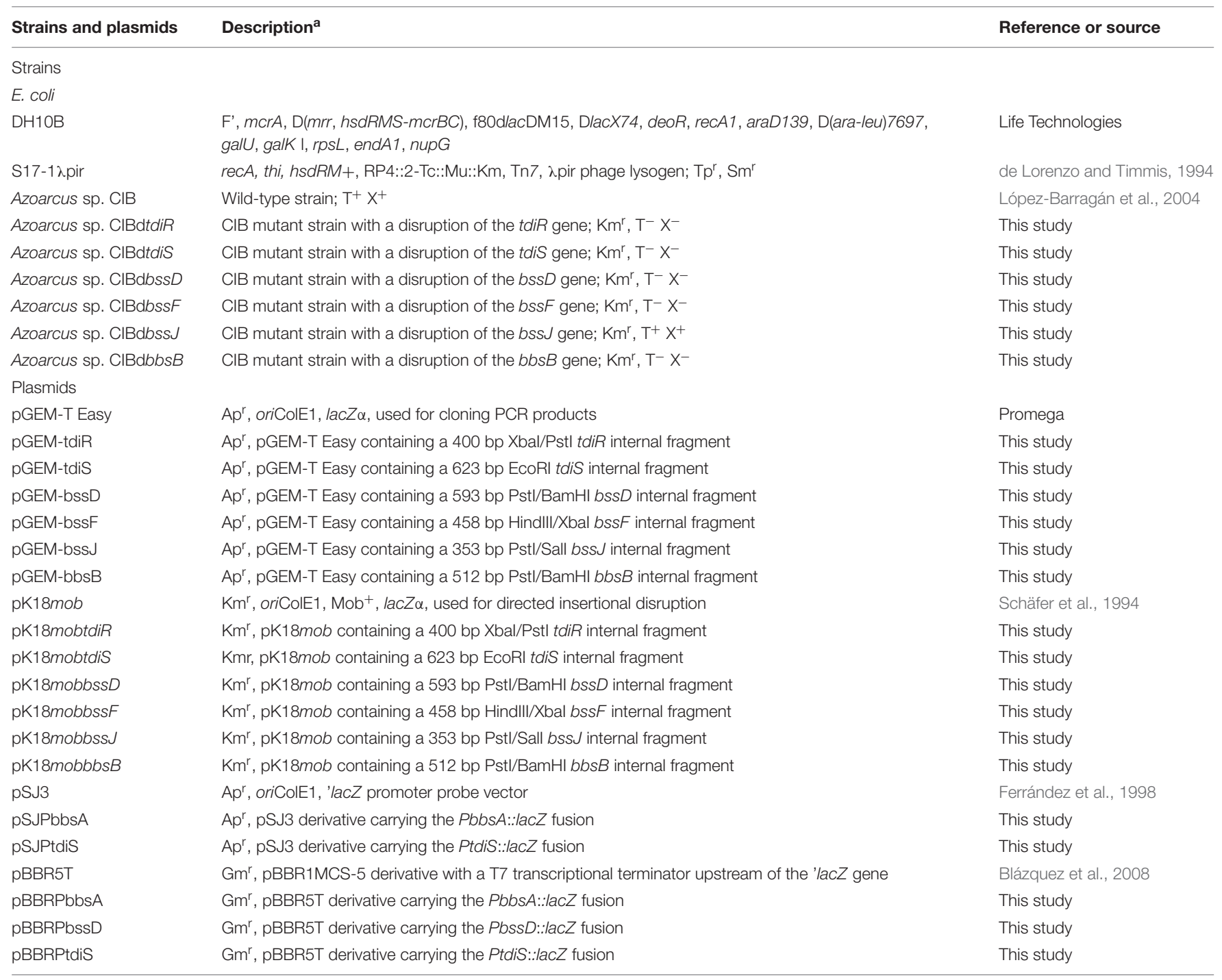

${ }^{a}$ The abbreviations used are: $T^{+}$, anaerobic growth in toluene; $X^{+}$, anaerobic growth in $m$-xylene; $T^{-}$, lack of anaerobic growth in toluene; $X^{-}$, lack of anaerobic growth in $\mathrm{m}$-xylene; $\mathrm{Ap}^{r}$, ampicillin-resistant; $\mathrm{Gm}^{r}$, gentamicin-resistant; $\mathrm{Km}^{r}$, kanamycin-resistant; $\mathrm{Sm}^{r}$, streptomycin-resistant.

pGEM-bssJ plasmid was PstI/SalI double-digested and the 347-bp fragment was then subcloned producing plasmid pK18mobbssJ (Table 1). For gene disruption of the bbsB gene, a $0.5-\mathrm{kb}$ internal fragment was PCR amplified using primers $5 \mathrm{BbsB}$ and $3 \mathrm{BbsB}$, and cloned in plasmid pGEM-bbsB (Table 1). The pGEM-bbsB plasmid was Pst I/BamHI double-digested and the 512-bp fragment was then subcloned producing plasmid pK18mobbbsB (Table 1). The derivative pK18mob plasmids

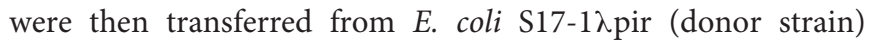
into Azoarcus sp. CIB (recipient strain) by biparental filter mating as previously described (de Lorenzo and Timmis, 1994; López-Barragán et al., 2004). Exconjugant Azoarcus sp. CIB mutant strains harboring the disrupted genes by insertion of the corresponding suicide plasmids, were isolated aerobically on kanamycin-containing MC medium lacking nitrate and containing $0.2 \%$ citrate as the sole carbon source for counter-selection of donor cells. The mutant strains were analyzed by PCR to confirm the disruption of the target genes.

\section{Construction of lacZ Translational Fusions}

To construct plasmid pSJPbbsA that harbors the PbbsA::lacZ translational fusion, a 356-bp XbaI/PstI DNA fragment containing the bbsA (AzCIB_4526)-orfB (AzCIB_4527) intergenic region was PCR-amplified from Azoarcus sp. CIB chromosomal DNA by using oligonucleotides 5PbbsA and 3PbbsA ( $5^{\prime}$-AACTG CAGGACATGACGCCTCCGCAGCATTTG-3' (Supplementary Table S1), and then cloned into the XbaI/PstI double-digested pSJ3 plasmid (Table 1). Plasmid pBBRPbbsA was constructed by subcloning a $3.5-\mathrm{kb}$ EcoRI/HindIII fragment harboring the PbbsA::lacZ translational fusion from pSJPbbsA into the EcoRI/HindIII double-digested broad-host range plasmid pBBR5T (Table 1). To construct plasmid pBBRPbssD that 
carries the PbssD::lacZ fusion, a 335-bp SpeI/BamHI DNA fragment containing the $t d i S-b s s D$ intergenic region was PCRamplified from Azoarcus sp. CIB chromosomal DNA by using oligonucleotides 5PtdiS and 3PbssD (Supplementary Table $\mathrm{S} 1$ ), and then cloned into the SpeI/BamHI double-digested pBBRPbbsA (Table 1). To construct plasmid pSJ3PtdiS that carries the PtdiS::lacZ translational fusion, a 314-bp BamHI fragment containing the bssD-tdiS intergenic region was PCR-amplified from Azoarcus sp. CIB chromosomal DNA by using oligonucleotides 3PbssD and PtdiS3'(Supplementary Table S1), and then cloned into the BamHI digested pSJ3 plasmid (Table 1). Plasmid pBBRPtdiS was constructed by subcloning a $3.5-\mathrm{kb}$ EcoRI/HindIII DNA fragment harboring the PtdiS::lacZ fusion from pSJ3PtdiS into the EcoRI/HindIII double-digested broad-host range plasmid pBBR5T (Table 1).

\section{RNA Extraction and Reverse Transcription-PCR Experiments}

Azoarcus sp. CIB cells grown in MC medium containing the appropriate carbon sources were harvested at the midexponential phase of growth and stored at $-80^{\circ} \mathrm{C}$. Azoarcus sp. $C I B d b b s B$ and $C I B d b s s D$ strains grown in kanamycin-containing MC medium with pyruvate plus toluene were harvested at the stationary phase of growth and stored at $-80^{\circ} \mathrm{C}$. Pellets were thawed, and cells were lysed in TE buffer $(10 \mathrm{mM}$ Tris- $\mathrm{HCl}$, $\mathrm{pH} 7.5,1 \mathrm{mM}$ EDTA) containing $50 \mathrm{mg} \mathrm{ml}^{-1}$ lysozyme. Total RNA was extracted using the RNeasy mini kit (Qiagen), including a DNase treatment according to the manufacturer instructions (Ambion), precipitated with ethanol, washed, and resuspended in RNase-free water. The concentration and purity of the RNA samples were measured by using a ND1000 Spectrophotometer (Nanodrop Technologies) according to the manufacturer's protocols. Synthesis of total cDNA was carried out with $20 \mu \mathrm{l}$ of reverse transcription reactions containing $400 \mathrm{ng}$ of RNA, $0.5 \mathrm{mM}$ concentrations of each dNTP, 200 U of SuperScript II reverse transcriptase (Invitrogen), and $5 \mu \mathrm{M}$ concentrations of random hexamers as primers in the buffer recommended by the manufacturer. Samples were initially heated at $65^{\circ} \mathrm{C}$ for $5 \mathrm{~min}$ then incubated at $42^{\circ} \mathrm{C}$ for $2 \mathrm{~h}$, and the reactions were terminated by incubation at $70^{\circ} \mathrm{C}$ for $15 \mathrm{~min}$. In standard $\mathrm{RT}$-PCR reactions, the cDNA was amplified with $1 \mathrm{U}$ of AmpliTaq DNA polymerase (Biotools) and $0.5 \mu \mathrm{M}$ concentrations of the corresponding primer pairs (oligonucleotides 1-20; Supplementary Table S1). Samples were initially denatured by heating at $94^{\circ} \mathrm{C}$ for $3 \mathrm{~min}$. A 30-cycle amplification program was followed $\left(94^{\circ} \mathrm{C}\right.$ for $40 \mathrm{~s}, 60^{\circ} \mathrm{C}$ for $40 \mathrm{~s}$ and $72^{\circ} \mathrm{C}$ for $60 \mathrm{~s}$ ). Control reactions in which reverse transcriptase was omitted from the reaction mixture ensured that DNA products resulted from the amplification of cDNA rather than from DNA contamination. The dnaE gene encoding the $\alpha$-subunit of DNA polymerase III was used to provide an internal control cDNA that was amplified with oligonucleotides 5'POLIIIHK/3'POLIIIHK (Supplementary Table S1). The expression of the internal control was shown to be constant across all samples analyzed.

\section{$\beta$-Galactosidase Assays}

The $\beta$-galactosidase activities from promoter-lac $Z$ reporter fusions were measured with permeabilized cells when cultures reached mid-exponential or stationary phase of growth, as described (Miller, 1972).

\section{Sequence Data Analyses}

Nucleotide sequence analyses were done at the National Center for Biotechnology Information (NCBI) server ${ }^{1}$. The amino acid sequences of the open reading frames were compared with those present in databases using the TBLASTN algorithm (Altschul et al., 1990) at the NCBI server ${ }^{2}$. Pairwise and multiple protein sequence alignments were made with the ClustalW program (Thompson et al., 1994) at the EMBL-EBI server ${ }^{3}$. Phylogenetic analysis of the different proteins was carried out according to the Kimura two-parameter method (Kimura, 1980), and a tree was reconstructed using the neighbor-joining method (Saitou and Nei, 1987) of the PHYLIP program (Felsenstein, 1993) at the TreeTop-GeneBee server ${ }^{4}$ and represented using TreeView X 0.5.1 (Glasgow University).

\section{RESULTS AND DISCUSSION}

\section{The bss-bbs Cluster Is Involved in the Anaerobic Degradation of Toluene and $m$-Xylene in Azoarcus sp. CIB}

As indicated in the Introduction, the in silico analysis of the genome sequence of Azoarcus sp. CIB revealed a gene cluster of 26 open reading frames most of which encode orthologous of the Bss and Bbs proteins involved in the peripheral pathway for the anaerobic degradation of toluene in several bacteria. This cluster is located in the close vicinity of the $m b d$ genes encoding the 3-methylbenzoyl-CoA anaerobic central pathway (Juárez et al., 2013), strongly suggesting that it constitutes the bss-bbs cluster for the anaerobic degradation of toluene and $m$-xylene in strain CIB (Zamarro et al., 2016) (Figure 1B).

Since the toluene peripheral pathway was shown to be inducible when bacteria grow in the presence of toluene (Coschigano, 2000; Achong et al., 2001; Hermuth et al., 2002; Kube et al., 2004; Kühner et al., 2005), we checked whether the expression of the $b s s-b b s$ genes was inducible when Azoarcus sp. CIB cells were grown anaerobically on toluene or $m$-xylene with respect to the use of succinate or benzoate as sole carbon source. The analysis of the RT-PCR amplification products revealed that the expression of the $b s s A$ and $b b s A$ genes is induced when the cells grow in the presence of toluene or $m$-xylene as sole carbon sources (Figures 3A,B), suggesting their participation in the anaerobic degradation of both aromatic hydrocarbons.

Usually the transcription of anaerobic degradation pathways is regulated by the presence of oxygen in the culture medium

\footnotetext{
${ }^{1}$ http://www.ncbi.nlm.nih.gov

${ }^{2}$ http://blast.ncbi.nlm.nih.gov

${ }^{3}$ http://www.ebi.ac.uk/Tools/msa/clustalw2/

${ }^{4}$ http://www.genebee.msu.su/services/phtree_reduced.html
} 


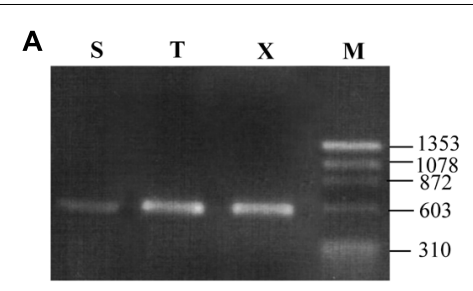

bss

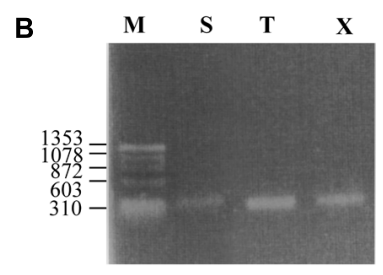

bbs

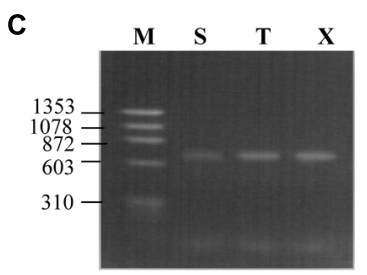

tdi

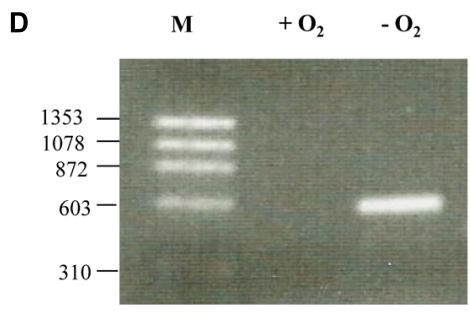

bss
E $\quad+\mathrm{O}_{2}-\mathrm{O}_{2}$

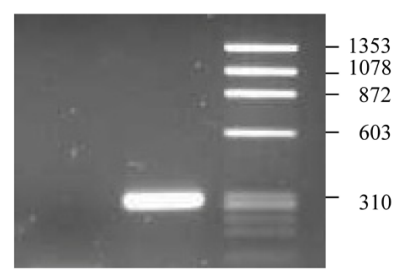

bbs

FIGURE 3 | The expression of the bss, bbs and tdi genes is inducible in Azoarcus sp. CIB. Agarose gel electrophoresis of the RT-PCR products. Gene expression was monitored by RT-PCR as described in Section "Materials and Methods" with the primer pairs bssA5new/bssA3new, bbsA331.3/bbsA5new and tdiRint5/tdiSF.3 (Supplementary Table S1) that amplify a bssA gene fragment (A,D), a bbsA gene fragment (B,E), or the tdiS-tdiR intergenic region (C), respectively. (A-C) Azoarcus sp. CIB cells were grown anaerobically until mid-exponential phase by using $0.2 \%$ succinate $(S)$, $250 \mathrm{mM}$ of toluene (T) or $250 \mathrm{mM} m$-xylene $(X)$ as sole carbon source. (D,E) Azoarcus sp. CIB cells were grown under anaerobic $\left(-\mathrm{O}_{2}\right)$ or aerobic $\left(+\mathrm{O}_{2}\right)$ conditions using toluene as only carbon source. Lanes M, molecular size markers (Haelll-digested $\Phi \times 174$ DNA); numbers indicate the sizes of the markers (in bp).

\section{A}

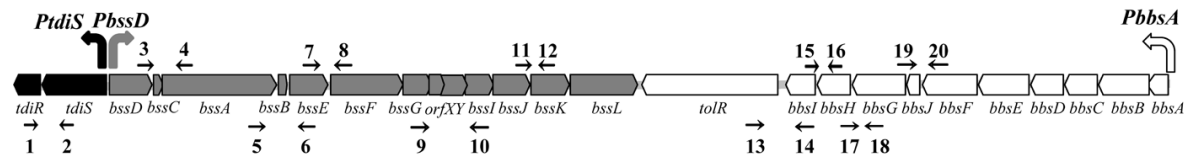

B

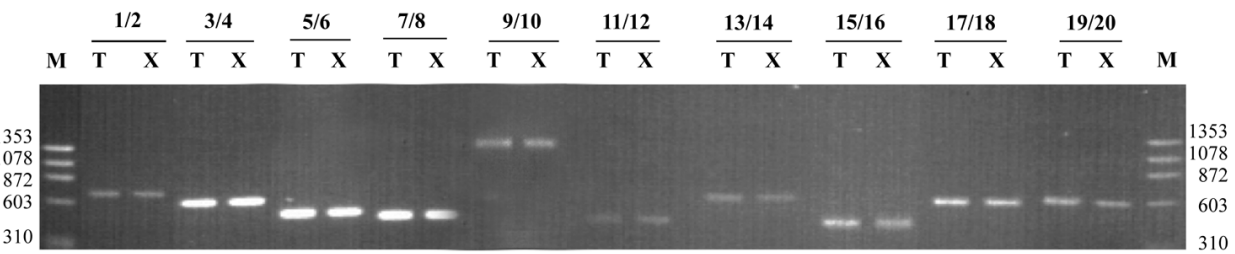

C

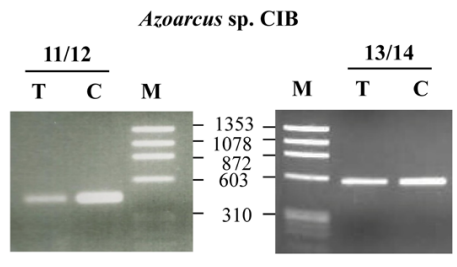

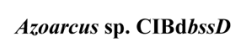

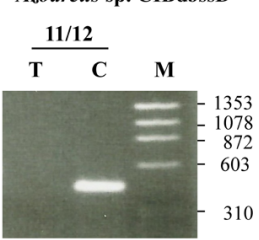

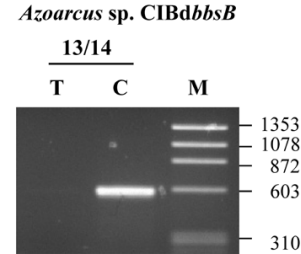

FIGURE 4 | Transcriptional organization of the tdi-bss-bbs genes: (A) Schematic representation of the tdi-bss-bbs gene cluster from Azoarcus sp. CIB. The regulatory tdi genes are indicated by black arrows; the catabolic bss and bbs genes are indicated by gray and white arrows, respectively. The PtdiS, Pbss $D$ and PbbsA promoters are represented by bent arrows. The intergenic regions whose expression was checked by RT-PCR are marked by the oligonucleotides (thin arrows) used in the analysis (1-20; see Supplementary Table S1). (B) Agarose gel electrophoresis of RT-PCR products. RT-PCRs from Azoarcus sp. CIB cells grown under denitrifying conditions on $250 \mathrm{mM}$ toluene (lanes T) or $250 \mathrm{mM} \mathrm{m}$-xylene (lanes X) were performed as described in Section "Materials and Methods." The numbers correspond to each primer pair that amplifies each of the intergenic regions indicated in (A). Lanes M, molecular size markers (Haelll-digested $\Phi$ X174 DNA); numbers indicate the sizes of the markers (in bp). (C) RT-PCRs from Azoarcus sp. CIB, Azoarcus sp. CIBdbssD and Azoarcus sp. CIBdbbsB cells anaerobically grown until stationary phase in $0.2 \%$ pyruvate plus $250 \mathrm{mM}$ toluene (lanes T) by using the oligonucleotide pairs 11/12 and 13/14 (Supplementary Table S1). Lanes C, PCRs performed with the same primer pairs and with genomic DNA as a positive control. Lanes M, molecular size markers (Haell-digested ФX174 DNA); numbers indicate the sizes of the markers (in bp). 
A

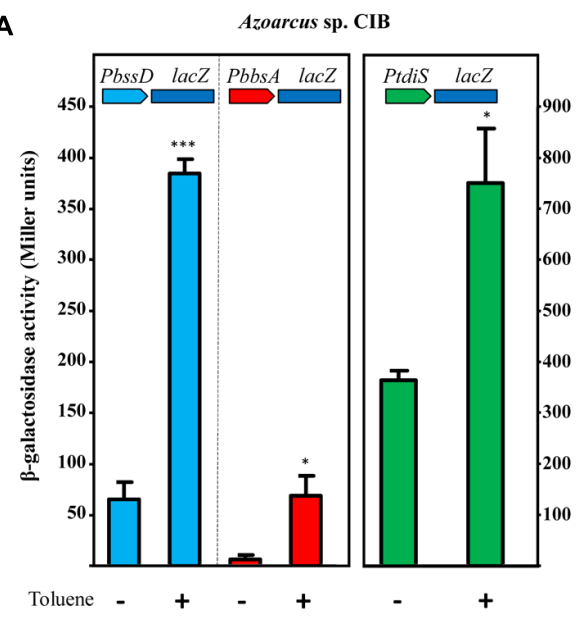

B

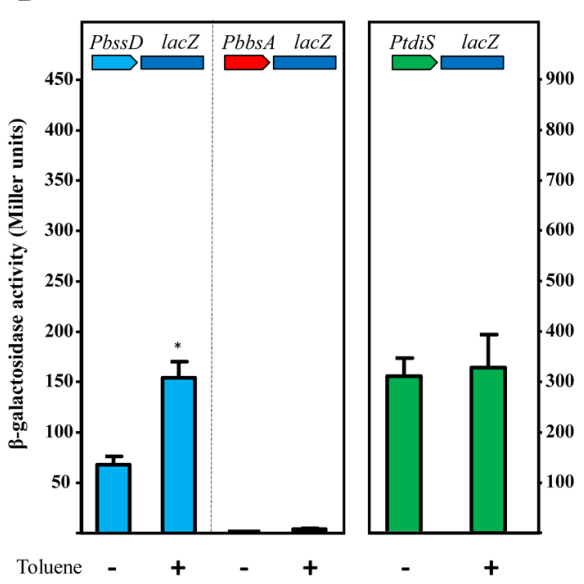

FIGURE 5 | TdiR is a transcriptional activator of the PbssD, PbbsA and PtdiS promoters. Cells of Azoarcus sp. CIB (A) or Azoarcus sp. ClBdtdiR (B) containing plasmids pBBRPbssD, pBBRPbbsA, or pBBRPtdiS that express the PbssD::lacZ, PbbsA::lacZ, or PtdiS::IacZ translational fusions, respectively, were grown anaerobically on $0.2 \%$ pyruvate $(-)$ or $0.2 \%$ pyruvate plus toluene $(+)$ until stationary growth phase. $\beta$-galactosidase activity values were determined as detailed in Section "Materials and Methods." Error bars represent standard deviation of three different experiments, and asterisks mark the results that are statistically significant (unpaired $t$-test; ${ }^{* * *} P$-value $<0.001,{ }^{*} P$-value $0.01-0.05$ ).

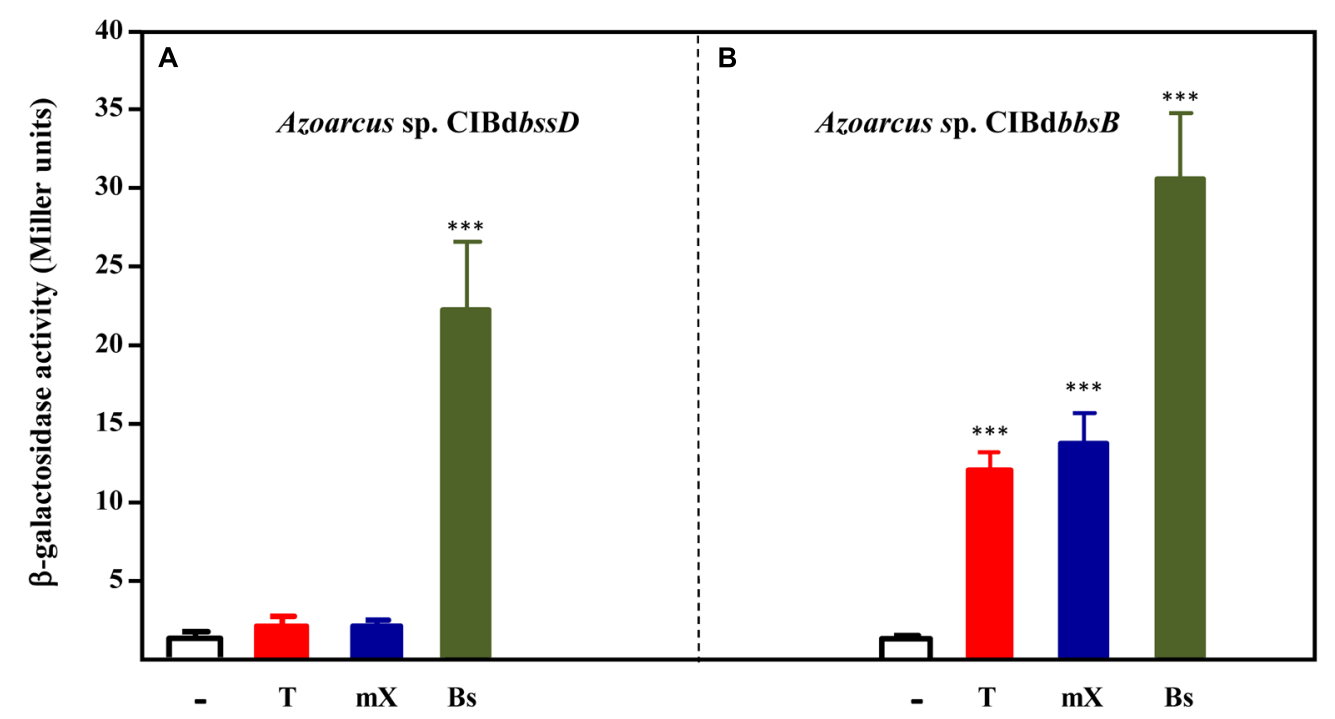

FIGURE 6 | Analysis of the inducers of the PbbsA promoter. Cells of Azoarcus sp. CIBdbssD (A) or Azoarcus sp. CIBdbbsB (B) containing plasmid pBBRPbbsA that expresses the PbbsA::lacZ translational fusion, were grown anaerobically until stationary growth phase in $0.2 \%$ pyruvate (-) or in $0.2 \%$ pyruvate plus toluene (T), $m$-xylene (mX), or benzylsuccinate (Bs). $\beta$-galactosidase activity values were determined as detailed in Section "Materials and Methods." Error bars represent standard deviation of three different experiments, and asterisks mark the results that are statistically significant (unpaired $t$-test; $* * * P$-value $<0.001$ ).

(Durante-Rodríguez et al., 2006; Carmona et al., 2009; Juárez et al., 2013). To determine if the bss-bbs cluster from Azoarcus sp. CIB is under oxygen control, gene expression studies were performed with cells grown with toluene as sole carbon source under aerobic or anaerobic conditions. RT-PCR experiments showed that the $b s s A$ and $b b s A$ genes are expressed only under anoxic conditions (Figures 3D,E). A similar oxygen-dependent expression of the bss genes was reported in Magnetospirillum sp. TS-6 (Shinoda et al., 2005), and it appears to be consistent with the fact that BSS is a strictly anaerobic enzyme (Beller and Spormann, 1998; Leuthner et al., 1998; Heider et al., 2016). Nevertheless, the oxygen-dependent regulation of the anaerobic toluene degradation pathway may differ from one strain to another since the bss genes from Thauera sp. DNT-1 were shown to be transcribed both in aerobic and anaerobic conditions (Shinoda et al., 2004).

To confirm that the bss-bbs cluster described above was responsible for the anaerobic degradation of toluene and $m$-xylene in Azoarcus sp. CIB, we constructed CIB mutant strains with insertional disruptions within some of the bss and $b b s$ genes 

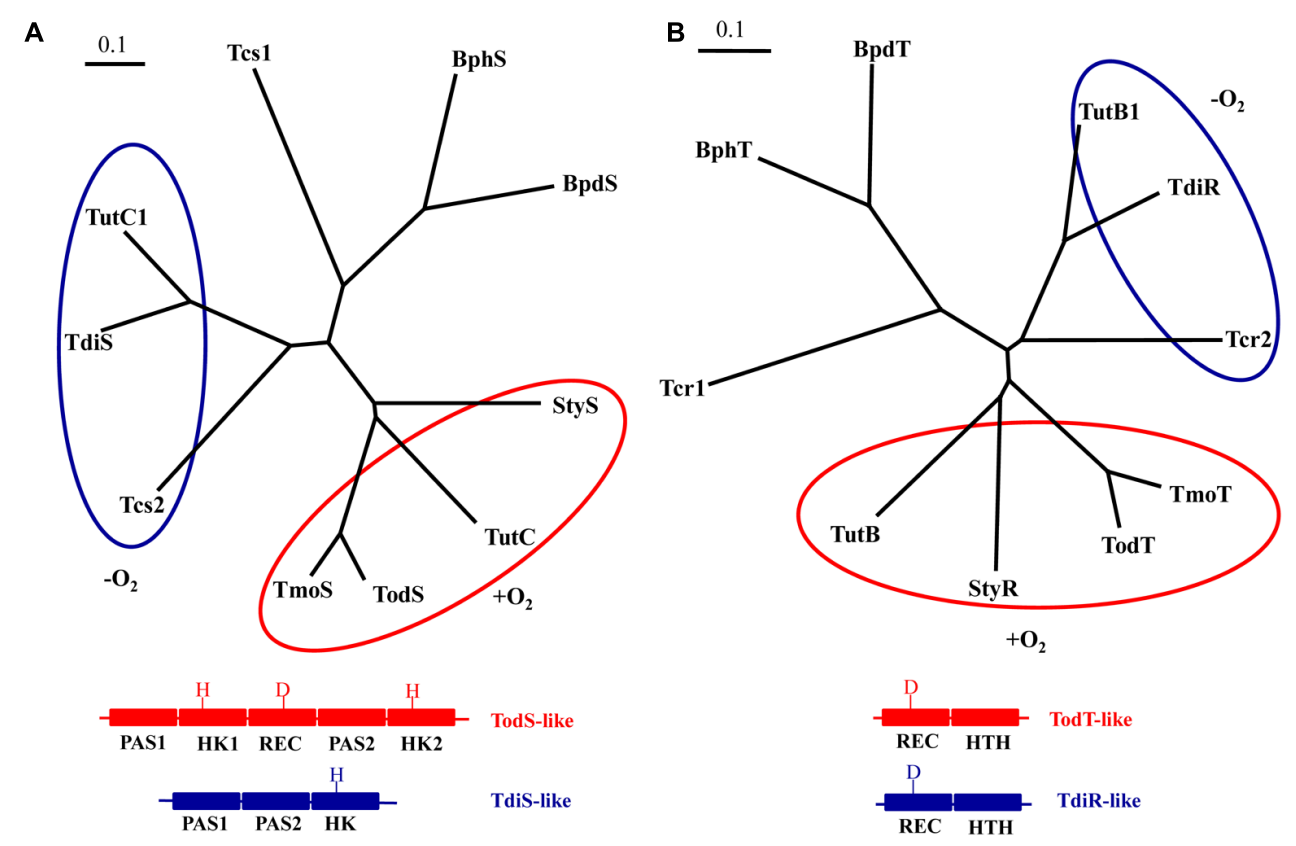

FIGURE 7| Phylogenetic relationships of the two-component regulatory systems involved in the regulation of aromatic hydrocarbon catabolic pathways: (A) Phylogenetic tree built from the multiple amino acid sequence alignment of the sensor histidine kinases TdiS from Azoarcus sp. CIB (ABK15651), TutC1 from T. aromatica T1 (AAD12187), Tcs2 from "A. aromaticum" sp. EbN1 (YP_158339), StyS from Pseudomonas sp. Y2 (CAA03998), TutC from P. mendocina (AAL13332), TodS from P. putida DOT-T1 (AAC45438), TmoS from P. mendocina (AAL13333), Tcs1 from A. aromaticum sp. EbN1 (YP_158337), BphS from Rhodococcus sp. RHA1 (BAC75411) and BpdS from Rhodococcus sp. M5 (AAB52543) using the program CLUSTALW and visualized with the TreeView software application. The aerobic TodS-like and anaerobic TdiS-like families are circled by red and blue lines, respectively, and a scheme of their different domain architecture and primary structure is shown at the bottom. PAS, HK, and REC correspond to the PAS sensor, autokinase and receiver domains, respectively. $\mathrm{H}$ and D indicate the presence of key phosphorylatable histidine and aspartic acid residues at the HK and REC domains, respectively. (B) Phylogenetic tree built from the multiple amino acid sequence alignment of the regulatory protein TdiR from Azoarcus sp. CIB (ABK15650), TutB1 from T. aromatica T1 (AAD12186), Tcr2 from "A. aromaticum" sp. EbN1 (YP_158340), TutB from T. aromatica T1 (AAD12186), StyR from Pseudomonas sp. Y2 (CAA03999), TodT from P. putida DOT-T1 (CAB43736), TmoT from P. mendocina (AAL13333), Tcr1 from A. aromaticum sp. EbN1 (YP_158338), BphT from Rhodococcus sp. RHA1 (BAC75412) and BpdT from Rhodococcus sp. M5 (AAB52544) using the program CLUSTALW and visualized with the TreeView software application. The aerobic TodT-like and anaerobic TdiR-like families are circled by red and blue lines, respectively, and a scheme of their similar domain architecture but different primary structure is shown at the bottom. REC and HTH correspond to the receiver and helix-turn-helix DNA-binding domains, respectively. D indicates the presence of key phosphorylatable aspartic acid residue at the REC domain. The bars represent one inferred amino acid substitution per ten amino acids.

that are orthologous to those that have been reported or suggested to be essential for toluene degradation in other bacteria. Thus, we constructed the Azoarcus sp. CIBdbssD, and CIBdbbsB mutant strains harboring the $b s s D$ and $b b s B$ disrupted genes, respectively (Table 1). As expected, none of the two mutant strains was able to use toluene as the sole carbon and energy source under anaerobic conditions (Table 1). Interestingly, none of the mutant strains was also able to use anaerobically $m$-xylene as carbon source (Table 1). Although inactivation of the bssA gene in Azoarcus sp. strain $\mathrm{T}$ was shown previously to prevent anaerobic growth on $m$-xylene (Achong et al., 2001), and (3-methyl)benzylsuccinate was proposed to be converted into 3-methylbenzoyl-CoA via a modified $\beta$-oxidation pathway (Figure 1A) (Krieger et al., 1999), a genetic demonstration that the $b b s$ genes were indeed needed for the anaerobic growth on $m$-xylene was still lacking. In fact, some studies had suggested the existence of separate Bbs isoenzymes in toluene and $m$-xylene catabolism (Leutwein and Heider, 2002). However, our results reveal that both the bss and $b b s$ genes are essential for the anaerobic degradation of toluene and $m$-xylene in Azoarcus sp. CIB.
So far, only the bssDCABE genes have been shown to be essential for the anaerobic conversion of toluene to benzylsuccinate (Leuthner et al., 1998; Krieger et al., 2001; Bhandare et al., 2006; Funk et al., 2014; Heider et al., 2016). However, the bss cluster contains additional genes of unknown function that are conserved in all toluene degraders, e.g., the $b s s F$ gene, or that are restricted to bss clusters from beta-proteobacteria, e.g., the bssGIJKL genes (Figure 2) (Kube et al., 2004; Carmona et al., 2009; Kim et al., 2014). To gain further knowledge on whether these additional bss genes might be essential for the initial reaction of the toluene/ $m$-xylene degradation pathway, we constructed Azoarcus sp. CIB mutant strains with disruptional insertions within the bss $F$ and $b s s J$ genes. Whereas the strain Azoarcus sp. CIBdbssF was unable to grow anaerobically in toluene or $m$-xylene as sole carbon sources, the strain Azoarcus sp. CIBdbss J could use these two aromatic hydrocarbons as growth substrates (Table 1). Therefore, these results reveal that inactivation of $b s s F$ prevents the anaerobic growth on toluene and $m$-xylene, and suggest that this gene and/or any of the downstream co-transcribed genes, i.e., bss $G$, 

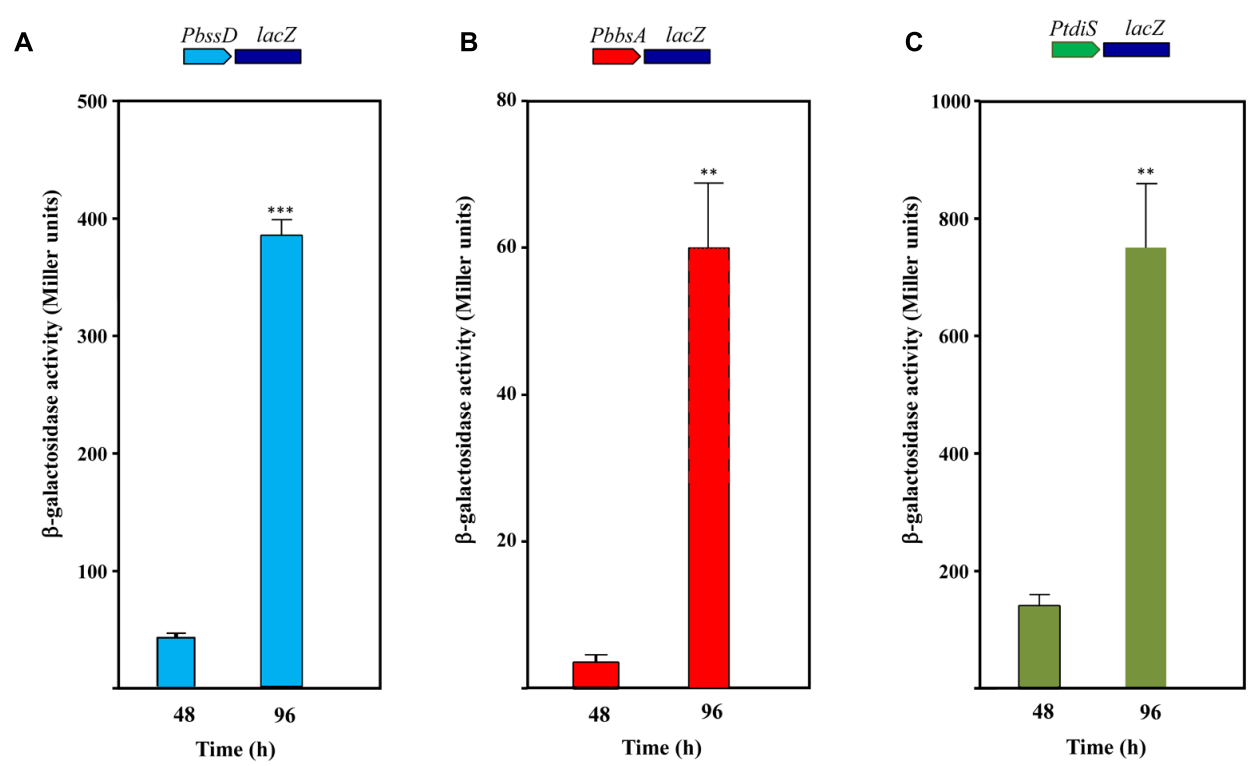

FIGURE 8 | Carbon catabolite control of the PbbsA, PbssD and PtdiS promoters in Azoarcus sp. CIB. Azoarcus sp. CIB cells containing plasmid pBBRPbssD (PbssD::lacZ fusion) (A), pBBRPbbsA (PbbsA::/acZ fusion) (B) or pBBRPtdiS (PtdiS::lacZ fusion) (C) were grown anaerobically in $0.2 \%$ pyruvate plus 250 mM toluene, and after $72 \mathrm{~h}$ they were supplemented with an additional amount of $10 \mathrm{mM}$ nitrate. Culture samples were collected at mid-exponential phase (48 $\mathrm{h}$ ) or at stationary phase (96 h). $\beta$-galactosidase activity values were determined as detailed in Section "Materials and Methods." Error bars represent standard deviation of three different experiments, and asterisks mark the results that are statistically significant (unpaired $t$-test; ${ }^{* * *} P$-value $<0.001,{ }^{* *} P$-value $0.001-0.01$ ).

orfXY, bssI (see below), are needed for the synthesis of a functional BSS enzyme. The BssF protein (580 amino acids) does not show significant similarity to other proteins of known function, which precludes so far any prediction of its functional role in the BSS activity. On the other hand, although the BssJ protein (307 amino acids) appears to be not essential for the BSS activity, we cannot rule out its involvement in toluene degradation by, for instance, mediating in the stress response caused by toxic aromatic hydrocarbons (Trautwein et al., 2008).

\section{Transcriptional Organization of the bss-bbs Gene Cluster}

The organization of the genes within the $b s s-b b s$ cluster in Azoarcus sp. CIB is very similar to that observed in other betaproteobacteria (Figure 2). The bss and bbs genes are arranged in two opposite orientations and most of the genes are separated by very short distances suggesting that they constitute two convergent operons (Zamarro et al., 2016) (Figure 4A). The existence of the bssDCABEFGH catabolic operon has been described in " $A$. aromaticum" EbN1 (Kube et al., 2004), but it is not known whether these genes are cotranscribed with the bssIJKLM genes. On the contrary, whereas the bss cluster from Azoarcus sp. strain T contains a bssDCABE operon and a transcriptional initiation site upstream of bss $F$ (Achong et al., 2001), the tutE (bssD) gene of $T$. aromatica $T 1$ constitutes an operon different to that formed by tutFDGH (bssCABE) (Coschigano, 2000). On the other hand, the transcriptional organization of the $b b s$ cluster has not been elucidated so far in any bacteria.
Since a transcriptional analysis of the whole gene cluster for anaerobic toluene and $m$-xylene degradation in bacteria has not been reported so far, we aimed to validate the presumed transcriptional organization of the $b s s-b b s$ genes in strain CIB. To this end, we performed RT-PCR experiments using total RNA harvested from Azoarcus sp. CIB cells grown anaerobically in toluene or $m$-xylene as sole carbon sources, and different primer sets that amplify $b s s D-b s s C(3 / 4), b s s B-b s s E(5 / 6), b s s E-b s s F(7 / 8)$, bssG-orfX-orfY-bssI (9/10), bssJ-bssK (11/12), tolR-bbsI (13/14), $b b s I-b b s H$ (15/16), bbsH-bbsG (17/18) and bbsJ-bbsF (19/20) gene fragments (Figure 4A). The results obtained (Figure 4B) strongly suggest that the $b s s D-L$ and bbsA-tol genes are cotranscribed and, therefore, constitute two separate convergent operons. To confirm that the bss and $b b s$ genes constitute two separate operons, we performed gene expression studies in the Azoarcus sp. CIBdbssD and CIBdbbsB mutant strains that harbor insertional disruptions within the $b s s D$ and $b b s B$ genes, respectively (Table 1). To this end, the two mutant strains were grown anaerobically in the presence of toluene, and RTPCR analyses revealed the lack of expression of the bss and bbs genes (Figure 4C), hence suggesting that the mutations caused polar effects on the expression of the genes located dowsntream of the insertion site and, therefore, that these genes are cotranscribed. Therefore, all these data taken together suggest that in Azoarcus sp. CIB the bssD-L and bbsA-tol genes are arranged as two separate convergent operons. A comparative analysis of the upstream regions of the $b s s D$ and $b b s A$ genes in different Azoarcus, Aromatoleum, Thauera, and Herminiimonas strains confirmed the existence of the previously proposed $P b s s D$ and $P b b s A$ promoters, respectively (Supplementary Figure S1). PbssD 
and PbbsA contain potential $-10 /-35$ sequences recognized by the RNA polymerase as well as the transcription initiation sites which have been mapped for bssD in T. aromatica (Coschigano, 2000; Hermuth et al., 2002; Kube et al., 2004) and Azoarcus sp. strain $\mathrm{T}$ (Achong et al., 2001), and for bbsA in T. aromatica K172 (Leuthner and Heider, 2000). Similar PbssD and PbbsA promoter regions driving the expression of the bss and bbs operons were identified in Azoarcus sp. CIB (Figure 4A and Supplementary Figure S1). Although an internal promoter or RNA processing event was reported upstream of $b s s C$ in some Thauera strains (Coschigano, 2000; Hermuth et al., 2002), the predicted stemloop structure resembling a RNase processing site (Hermuth et al., 2002) is not observed in the bss operon of Azoarcus sp. CIB.

A couple of conserved genes, i.e., tdiS (tutC1) and tdiR (tutB1), are located upstream of the bss genes in most anaerobic toluene degraders from the beta-proteobacteria group (Figure 2). These two genes were proposed to code for a two-component regulatory system that regulates transcription of the catabolic bss genes (Coschigano, 2000; Achong et al., 2001; Hermuth et al., 2002; Kube et al., 2004; Kühner et al., 2005). A couple of orthologous $t d i S R$ genes are also divergently transcribed with respect to the $b s s$ genes in Azoarcus sp. CIB (Figures 1B, 2, 4A). RT-PCR studies revealed that $t d i S$ and $t d i R$ are co-transcribed (Figure $4 \mathbf{B}$ ) and, therefore, constitute an operon controlled by the PtdiS promoter in Azoarcus sp. CIB (Figure 4A). Moreover, the tdiSR operon appears to be slightly inducible when the cells grow anaerobically in toluene or $m$-xylene (Figure 3C), thus suggesting a common regulatory mechanism for the catabolic (bss, bbs) and predicted regulatory (tdiSR) genes in strain CIB.

All these results taken together constitute the first transcriptional analysis of the whole gene cluster responsible for the anaerobic peripheral pathway for toluene and $m$-xylene degradation in a single bacterial strain. In Azoarcus sp. CIB these genes are organized in two catabolic operons, bssDCABEFGIJJKL and $b b s A B C D E F J G H t o l$, and a putative regulatory operon, tdiSR, that are inducible when cells grow in toluene and $m$-xylene.

\section{tdiSR Control the Expression of the bss-bbs Genes in Azoarcus sp. CIB}

To confirm the implication of the tdiSR genes in the anaerobic degradation of toluene and $m$-xylene in Azoarcus sp. CIB, we constructed two mutant strains, Azoarcus sp. CIBdtdiS and Azoarcus sp. CIBd $t d i R$, that harbor disruptional insertions within the $t d i S$ (allows expression of $t d i R$ from the kanamycin resistance gene, see Supplementary Figure S2) and $t d i R$ genes, respectively. The two mutant strains were unable to grow using toluene or $m$-xylene as sole carbon sources (Table 1), thus suggesting that the $t d i S R$ genes are involved in the anoxic degradation of toluene/ $m$-xylene and they are likely behaving as an activator system of the $P b s s D$ and/or PbbsA promoters.

To study further the role of TdiSR in the activation of the PbssD and PbbsA promoters in Azoarcus sp. CIB, we constructed plasmids pBBRPbssD and pBBRPbbsA that contain the PbssD::lacZ and PbbsA::lacZ translational fusions, respectively (Table 1). The Azoarcus sp. CIB strains containing plasmid $\mathrm{pBBRPbssD}$ or pBBRPbbsA were grown anaerobically in the absence or presence of toluene or $m$-xylene, and $\beta$-galactosidase assays were performed at the end of the growth curve. As shown in Figure 5A, the presence of toluene increased the activity of the PbssD and PbbsA promoters by more than fivefold with respect to the activity observed in the absence of toluene (similar results were obtained with $m$-xylene, data not shown). These results are in agreement with the observed induction of the bss and bbs genes when Azoarcus sp. CIB grows anaerobically in toluene (Figures 3A,B). Moreover, the activity of the PbssD promoter appears to be significantly higher than that of the PbbsA promoter both in the absence or presence of toluene (Figure 5A). We then analyzed the activity of the $P b s s D$ and PbbsA promoters in the Azoarcus sp. CIBdtdiR mutant strain harboring the pBBRPbssD or pBBRPbbsA plasmids. In contrast to the wild-type strain, the Azoarcus sp. CIBdtdiR (pBBRPbbsA) strain did not show $\beta$-galactosidase activity even in the presence of toluene (Figure 5B), which strongly suggests that TdiR acts as an essential activator of the PbbsA promoter. On the other hand, the Azoarcus sp. CIBdtdiR (pBBRPbssD) strain revealed that the activity of the $P b s s D$ promoter region in the absence of toluene did not increase as much as in the wild-type strain in the presence of toluene (Figure 5B), suggesting that although TdiR is needed for a full induction of bss genes, there is some TdiR-independent activation of $P b s s D$ when the cells are in the presence of toluene.

Since the expression of the tdiSR genes is also induced when Azoarcus sp. CIB grows in the presence of toluene (Figure 3C), we checked whether the tdiR gene product could also act as a transcriptional activator of its own PtdiS promoter. To accomplish this, we constructed plasmid pBBRPtdiS, that contains the PtdiS::lacZ translational fusion (Table 1), and monitored the $\beta$-galactosidase activity in Azoarcus sp. CIB (pBBRPtdiS) and Azoarcus sp. CIBdtdiR (pBBRPtdiS) strains grown anaerobically in the absence or presence of toluene. Toluene increased the activity of the PtdiS promoter by more than twofold with respect to the activity observed in the absence of toluene in the wild-type but not in the $t d i R$ mutant strain (Figure 5).

All these results taken together reveal for the first time that the tdiSR genes, which are highly conserved in the anaerobic toluene degradation clusters of beta-proteobacteria, encode an activator system of the promoters that drive expression of the $b s s$ and $b b s$ catabolic genes and of that controlling transcription of the $t d i$ regulatory genes in the presence of toluene. Whereas the expression of the $b b s$ genes is strictly dependent on TdiR, the induction of the bss genes appears to be controlled by TdiR and some additional factors that respond to the presence of toluene in Azoarcus sp. CIB, and whose characterization requires further research.

\section{Benzylsuccinate Might Be the Inducer Molecule of the TdiSR-Mediated Control}

As described above, the expression of the tdi-bss-bbs genes is inducible in Azoarcus sp. CIB cells growing in the presence of toluene and $m$-xylene (Figures 3,4 ). In $T$. aromatica $\mathrm{T} 1$, benzylsuccinate or any other further intermediate in the toluene peripheral pathway (Figure 1A), was suggested 
to be the inducer of the tut(bss) genes (Coschigano and Bishop, 2004). To determine if toluene and/or $m$-xylene, or an intermediate metabolite derived from the anaerobic degradation of the former, are the true inducers of the TdiSRmediated control of the peripheral pathway in Azoarcus sp. $\mathrm{CIB}$, we analyzed the expression of the PbbsA::lacZ fusion in different CIB strains. We selected CIB mutants strains that lack: (i) the first enzymatic step for the conversion of toluene/m-xylene to benzylsuccinate/(3-methyl)benzylsuccinate, i.e., Azoarcus sp. CIBdbssD, which does not express the bss operon (Figure 4C); (ii) the modified $\beta$-oxidation pathway that converts benzylsuccinate/(3-methyl)benzylsuccinate to the central intermediates benzoyl-CoA/3-methylbenzoylCoA (Figure 1), i.e., Azoarcus sp. CIBdbbsB, which does not express the bbs operon (Figure 4C). Azoarcus sp. CIBdbssD (pBBRPbbsA) cells grown anaerobically on pyruvate or pyruvate supplemented with toluene or $m$-xylene as inducers showed very low $\beta$-galactosidase activity in all the tested conditions, thus suggesting that none of the two aromatic hydrocarbons is the real inducer of the $b b s$ operon (Figure 6A). On the contrary, Azoarcus sp. CIBdbbsB (pBBRPbbsA) cells, which retain a functional bss operon and should be capable to transform toluene and $m$-xylene accumulating benzylsuccinate and (3methyl)benzylsuccinate, respectively, presented $\beta$-galactosidase activity when they were cultivated anaerobically in the presence of toluene or $m$-xylene (Figure 6B). Therefore, all these data suggest that benzylsuccinate and (3-methyl)benzylsuccinate, rather than toluene and $m$-xylene, are the real inducers of the $b b s$ genes. In agreement with this hypothesis, the two Azoarcus sp. CIB mutant strains grown anaerobically in the presence of benzylsuccinate showed activation of the PbbsA promoter (Figure 6). Interestingly, none of Azoarcus strains could use benzylsuccinate as sole carbon and energy source, suggesting a poor uptake of this compound inside the cells.

Taken together all these results reveal that benzylsuccinate is the inducer compound of the TdiSR-mediated control of the toluene peripheral pathway in Azoarcus sp. CIB. Although a similar regulatory scenario can be predicted in other toluene degrader denitrifying bacteria, such as in Thauera strains (Coschigano and Bishop, 2004), benzylsuccinate may not always behave as the inducer molecule. Thus, it was reported that "A. aromaticum" EbN1 strain grown in a mixture of pyruvate and benzylsuccinate did not induce the expression of bss $A$ or the formation of Bss/Bbs proteins (Kühner et al., 2005). Nevertheless, the lack of induction of benzylsuccinate in strain EbN1 could be due to unefficient transport of this molecule or to putative pyruvate-dependent catabolite repression of the bss-bbs genes, as it was shown in strain CIB (see below), and therefore further studies should be carried out to confirm whether benzylsuccinate behaves also as an inducer molecule in "A. aromaticum" EbN1.

Two-component regulatory systems that consist of a sensor histidine kinase and its cognate transcriptional regulator have been shown to be involved in the control of the catabolism of aromatic hydrocarbons in several bacteria (Carmona et al., 2008). According to their primary structure and molecular architecture, the sensor histidine kinases of these two-component regulatory systems can be classified in at least three different phylogenetic groups, i.e., the TodS, TdiS and BphS groups (Figure 7A). Hybrid histidine kinases of the TodS family are complex enzymes that contain two sensor PAS domains, i.e., PAS1, that recognizes aromatic hydrocarbons (Busch et al., 2007), and PAS2, involved in dimerization (Koh et al., 2016), two transmitter (autokinase) domains, and a response regulator receiver domain (Figure 7A) (Busch et al., 2009). They are involved in the control of the genes for the aerobic degradation of toluene in Pseudomonas putida (TodS) (Busch et al., 2007), P. mendocina (TmoS) (SilvaJiménez et al., 2012) and, probably, in T. aromatica (TutC) (Leuthner and Heider, 1998), and styrene in some Pseudomonas strains (StyS) (Velasco et al., 1998; Leoni et al., 2003). Histidine kinases of the TdiS group are shorter than TodS-like kinases. They contain two tandem PAS domains, PAS1 and PAS2, and a single transmitter domain (Figure $\mathbf{7 A}$ ), and are encoded in the clusters for the anaerobic degradation of toluene in some strains of the genera Azoarcus/"Aromatoleum" (TdiS) (Achong et al., 2001); Thauera (TdiS/TutC1) (Leuthner and Heider, 1998) and Herminiimonas (Figure 2), as well as in the cluster for the anaerobic degradation of ethylbenzene in "A. aromaticum" sp. EbN1 (Tcs2) (Kühner et al., 2005). On the other hand, the large histidine-kinases BphS and BpdS from Rhodococcus sp. RHA1 and Rhodococcus sp. M5, respectively, are involved in the aerobic degradation of biphenyls and they harbor a $\mathrm{N}$-terminal domain similar to that of serine/threonine kinases and a C-terminal histidine-kinase domain (Takeda et al., 2010). It is worth noting that the amino acid residues shown to be involved in the binding to the inducer in the PAS1 domain of TodS, i.e., Phe46, Ile74, Phe79 and Ile114, are conserved in PAS1 domains of other TodS family members but not in any of the PAS domains of TdiS-like proteins. This observation is in agreement with the fact that TodS family members recognize aromatic hydrocarbons as inducers (Busch et al., 2007; Busch et al., 2009; Koh et al., 2016) but TdiS-like histidine kinases may respond to an intermediate, i.e., a benzylsuccinate derivative, rather than to the precursor aromatic hydrocarbon.

The response regulators of the two component regulatory systems that control the catabolism of aromatic hydrocarbons show a similar modular architecture with a $\mathrm{N}$-terminal receiver domain that contains the phosphoaccepting Asp residue (putative Asp58 in TdiR) and a C-terminal helix-turn-helix (HTH) DNA-binding domain of the NarL/FixJ family separated by a glutamine-rich Q linker (Figure 7B) (Milani et al., 2005). As with the sensor histidine kinases, three different phylogenetic groups can be identified when comparing their cognate response regulator partners, i.e., the TodT, TdiR and BphT groups (Figure 7B). The 3D-structure of the HTH domain of StyR shows four $\alpha$ helices, one of them responsible of DNA recognition with three residues (Lys175, Val176 and His179) putatively involved in the interaction with the operator region of the cognate promoter (Milani et al., 2005). The same amino acid residues are conserved in other StyR-like regulators, such as TodT, TutB and TmoT, all of which may recognize the consensus ATAAACN ${ }_{4}$ GTTTAT sequence at their corresponding operator regions (Lacal et al., 2006; Silva-Jiménez et al., 2012). Interestingly, these residues are not conserved in the HTH 
domains of the anaerobic TdiR-like response regulators, which were proposed to recognize a different palindromic region, GGTGTTCGCACC, that is conserved upstream of the -35 regions at the $P b s s D$ and $P b b s A$ promoters of different anaerobic toluene degraders (Supplementary Figure S1) (Kube et al., 2004).

In $T$. aromatica $\mathrm{T} 1$ the genes tutC1 (tdiS) and tutB1 (tdiR) are separated from the tutEFDGH (bssDCABE) catabolic genes by $t u t B$ and $t u t C$ (Figure 2). The products of the $t u t B$ and tut $C$ genes show high identity with TodS and TodT regulators, respectively, rather than with $\mathrm{TdiS}$ and $\mathrm{TdiR}$ regulators (Figure 7 ), thus strongly suggesting that TutBC are involved in the aerobic catabolism of toluene in T. aromatica T1 (Leuthner and Heider, 1998). However, the observation that a mutation in the tut $B$ gene disabled the capacity to grow anaerobically in toluene (Coschigano and Young, 1997) and reduced significantly the expression of the bss genes (Coschigano and Bishop, 2004), might suggest the existence of some cross-talk in the regulation of the aerobic and anaerobic toluene degradation pathways in T. aromatica $\mathrm{T} 1$.

\section{Carbon Catabolite Repression of the Toluene/m-Xylene Peripheral Pathway in Azoarcus sp. CIB}

The expression of the genes responsible of the catabolism of aromatic compounds is usually under carbon catabolite control when the cells grow in the presence of preferred carbon sources. This catabolite repression phenomenon has been extensively studied in the aerobic catabolism of aromatic compounds (Marqués et al., 1994; Cases et al., 1996; Carmona et al., 2008; Rojo, 2010), and it has also been described for the central pathways involved in the anaerobic catabolism of aromatic compounds in T. aromatica (Heider et al., 1998) and Azoarcus sp. CIB (López-Barragán et al., 2004; Carmona et al., 2009; Juárez et al., 2013; Valderrama et al., 2014). However, there were no reports about the expression of the peripheral pathways for anaerobic degradation of aromatic hydrocarbons when bacteria grow in the presence of alternative carbon sources.

To determine if the expression of the toluene/m-xylene anaerobic peripheral pathway in Azoarcus sp. CIB is under catabolite repression, we checked the activation of the $P b b s A$ and PbssD promoters in Azoarcus sp. CIB (pBBRPbbsA) and Azoarcus sp. CIB (pBBRPbssD) cells, respectively, grown in minimal medium containing toluene plus an additional carbon source, e.g, pyruvate, glutamate, glutarate or benzoate. All the carbon sources tested produced an inhibitory effect on the induction of $P b b s A$ and $P b s s D$ promoters, but this inhibition disappeared when the cells reached the stationary phase of growth and consumed the preferred carbon source (Figures $\mathbf{8 A}, \mathbf{B}$ ). In contrast, the induction of these promoters was observed at the exponential and stationary growth phases when the cells were grown in toluene as sole carbon source (Supplementary Figure S3). These results suggest that the bss and bbs genes are under carbon catabolite repression, but induction of these genes can be observed at the stationary growth phase as reported above (Figures 5, 6). Interestingly, we also observed a pyruvate-dependent catabolite repression of the PtdiS promoter when Azoarcus sp. CIB (pBBRPtdiS) cells were grown in minimal medium containing toluene plus pyruvate, and this repression was alleviated at the stationary phase of growth (Figure 8C). Thus, catabolite repression of the $t$ diST regulatory operon might be a major reason of the observed repression by organic acids of the TdiSR-dependent expression of the bss-bbs-genes in Azoarcus sp. CIB. Although carbon catabolite repression by organic acids had been reported in the aerobic catabolism of toluene in some bacteria (Duetz et al., 1996; Ruíz et al., 2004; Busch et al., 2010), catabolite repression of the peripheral pathway for the anaerobic degradation of toluene has not been shown before.

\section{Evolutionary Considerations on the Toluene Peripheral Pathway}

A detailed sequence comparison analysis of the organization of the $b s s$ - $b b s$ genes in different beta-proteobacteria revealed the existence of a region that shows a high variability among strains. Thus, the $b s s G$-bssI intergenic region contains a $b s s H$ gene encoding a putative transporter in " $A$. aromaticum" EbN1, but this gene is lacking in Herminiimonas sp. $\mathrm{CN}$, and is substituted

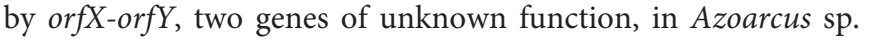
$\mathrm{CIB}$ and $A$. toluclasticus MF63 strains (Figure 2). Interestingly, this region divides the $b s s-b b s$ cluster of the CIB and MF63 strains into two different DNA fragments according to their GC content, i.e., a fragment containing the bssDCABEFG genes and whose GC content (58\%) is lower that than of the fragment containing the rest of the bss (bssIJKL) and bbs genes, whose GC content (65\%) matches the average GC content of the Azoarcus genome. It is worth noting that the bssDCABE genes from Azoarcus sp. T, T. aromatica T1 and Thauera sp. DNT1 have also a GC content $(<57 \%)$ lower than the average GC content (65\%) described for Azoarcus/Thauera strains (Liu et al., 2013; Martín-Moldes et al., 2015). On the contrary, the GC content of the bssDCABE genes from "A. aromaticum" EbN1, T. aromatica K172 and Magnetospirillum sp. TS-6 is close to the average GC content of the corresponding species. These observations suggest that the bssDCABEFG genes from Azoarcus sp. CIB, Azoarcus sp. T, T. aromatica T1 and Thauera sp. DNT-1 have an evolutionary origin different than that of their orthologous from Magnetospirillum sp. TS-6, "A. aromaticum" EbN1 and T. aromatica K172, and most probably they come from a microorganism, such as a Hermimiimonas-related strain, with a GC content lower than that of Thauera or Azoarcus strains. Interestingly, this subgrouping of Bss orthologs has also a reflect in the subtle differences observed in their reaction mechanism when using stable isotope tools (Kummel et al., 2013).

Analysis of the flanking regions of the $t d i-b s s$ - $b b s$ cluster in Azoarcus sp. CIB revealed the presence of full or truncated mobile genetic elements (Figure 2). Thus, next to the tdiSR genes there is a sequence $(\triangle i s t A)$ encoding the first amino acids of a truncated IS 21 transposase. ist $A /$ ist $B$ genes encoding the two subunits of a IS21 transposase are located adjacent to the bbs genes in "A. aromaticum" EbN1 (Figure 2) (Kube et al., 2004). On the other hand, next to the $b b s$ operon of strain CIB there is a set of genes $(\operatorname{orf} B-E)$ (Figure 2) that are orthologous to $c 1 A 68, c 1 A 87, c 1 A 90$, and $c 1 A 92$ genes that encode proteins of unknown function and are located 
within the cluster for anaerobic degradation of ethylbenzene in "A. aromaticum" EbN1 strain (Rabus et al., 2002). These genes are also present in a similar organization next to the bbs genes in A. toluclasticus MF63 (Figure 2). Downstream of the orfB-E genes in strain CIB there are three additional genes encoding two complete and one truncated IS4 transposase (Figure 2). All these observations strongly suggest that the $b s s$-bbs genes in Azoarcus sp. CIB and some closely related bacteria have been acquired by horizontal gene transfer events as a result of different genetic rearrangements. A different genetic organization is observed in the $b s s$-bbs clusters from phylogenetically distant delta-proteobacteria toluene-degrading obligate anaerobes, e.g., Geobacter or Desulfobacula (Figure 2) (Carmona et al., 2009; Wöhlbrand et al., 2013). The different genetic organization of the toluene peripheral pathway in facultative and obligate anaerobes correlates also with the significant differences found both in the predicted regulatory genes, e.g., TetR-like and XylR-like regulators are present in obligate anaerobes versus TdiSR-like regulators present in facultative anaerobes (Figure 2), and in the catabolic genes, e.g., bss and genes involved in auxiliary functions for Bss and Bbs enzymes are also distinct in facultative and strict anaerobes (Figure 2) (Wöhlbrand et al., 2013; Bozinovski et al., 2014). In summary, despite the biochemical strategy to convert toluene into benzoyl-CoA via benzylsuccinate appears to be shared by most anaerobic toluene-degraders, the complete set of genetic determinants involved in this peripheral pathway may have arisen through different evolutionary events in facultative and obligate anaerobes.

\section{CONCLUSION}

Toluene and $m$-xylene are important contaminant hydrocarbons that tend to accumulate in subsurface anoxic environments. We have shown here that the bss and bbs genes are essential for the anaerobic degradation of toluene and $m$-xylene in Azoarcus sp. CIB. Moreover, we have characterized for the first time the transcriptional organization and regulation of a complete cluster encoding the peripheral pathway for the anaerobic degradation of toluene and $m$-xylene in bacteria. Benzylsuccinate and (3-methyl)benzylsuccinate were shown to be the inducer molecules recognized by the TdiSR two-component regulatory system that specifically controls the activation of the $b s s, b b s$ and $t d i$ operons in Azoarcus sp. CIB. In addition to the TdiSR-mediated specific control, the expression of the bss and bbs genes in Azoarcus sp. CIB is under an overimposed regulation that depends on certain environmental factors, such as the presence/absence of oxygen or the availability of preferred carbon sources (catabolite repression). Interestingly, our results indicate that the bss and bbs operons from Azoarcus sp. CIB display differential regulation in the presence of toluene. Whereas the activity of the PbbsA promoter is strictly dependent on TdiR, the activation of the $P b s s D$ promoter is also under control of unknown factor(s) triggered by the presence of the aromatic hydrocarbons. Moreover, it is worth noting that the $P b s s D$ promoter shows a significant basal activity when the cells grow in the absence of toluene/m-xylene. This basal activity and the TdiR-independent activation of $P b s s D$ by toluene could be a strategy to increase the organism's capacity to react quickly to transient toluene availability. Thus, the presence of basal levels of BSS enzyme in the cell will facilitate the formation of some inducer intermediate (benzylsuccinate) and, therefore, the subsequent full induction of all regulatory and catabolic genes when the cells face the presence of the aromatic hydrocarbons. Our results are in agreement with recent reports which suggest that $b s s A$ transcription may occur at a basal level even in the absence of toluene, supporting the idea that detection of bssA gene transcripts alone is not sufficient to indicate toluene degradation activity in contaminated environments (Brow et al., 2013; Lünsmann et al., 2016). In this context, and for a reliable assessment of microbial activity in toluene-contaminated samples, we propose here to monitor the expression of the tightly regulated bbs genes as an alternative or complementary approach to the current methods based only on the study of bss $A$ expression. In this sense, highly conserved nucleotide sequence regions within the $b b s A B$ genes of different bacteria could be used to design degenerate oligonucleotides primers for amplification of a $b b s$ gene probe. Finally, the TdiSR-PbbsA regulatory couple identified in this work might constitute also an interesting genetic tool to develop whole cell biosensors for detecting benzylsuccinate, a widely used metabolic biomarker of in situ anaerobic bioremediation of toluene-contaminated sites (Young and Phelps, 2005).

\section{AUTHOR CONTRIBUTIONS}

$\mathrm{BB}$ carried out the practical work. BB, MC, and ED designed the experiments, analyzed the results, and wrote the manuscript.

\section{FUNDING}

This work was supported by grants BIO2012-39501, BIO201679736-R, and PCIN-2014-113 from the Ministry of Economy and Competitiveness of Spain and by a grant of Fundación Ramón Areces XVII CN. We acknowledge support of the publication fee by the CSIC Open Access Publication Support Initiative through its Unit of Information Resources for Research (URICI).

\section{ACKNOWLEDGMENTS}

We thank A. Valencia for technical assistance and Secugen S. L. for DNA sequencing.

\section{SUPPLEMENTARY MATERIAL}

The Supplementary Material for this article can be found online at: https://www.frontiersin.org/articles/10.3389/fmicb. 2018.00506/full\#supplementary-material 


\section{REFERENCES}

Achong, G. R., Rodriguez, A. M., and Spormann, A. M. (2001). Benzylsuccinate synthase of Azoarcus sp. strain T: cloning, sequencing, transcriptional organization, and its role in anaerobic toluene and $m$-xylene mineralization. J. Bacteriol. 183, 6763-6770. doi: 10.1128/JB.183.23.6763-67 70.2001

Altschul, S., Gish, W., Miller, W., Myers, E., and Lipman, D. (1990). Basic local alignment search tool. J. Mol. Biol. 215, 403-410. doi: 10.1016/S0022-2836(05) 80360-2

Beller, H. R., and Spormann, A. M. (1998). Analysis of the novel benzylsuccinate synthase reaction for anaerobic toluene activation based on structural studies of the product. J. Bacteriol. 180, 5454-5457.

Bertani, G. (1951). Studies on lysogenesis. I. The mode of phage liberation by lysogenic Escherichia coli. J. Bacteriol. 62, 293-300.

Bhandare, R., Calabroa, M., and Coschigano, P. W. (2006). Site-directed mutagenesis of the Thauera aromatica strain T1 tutE tutFDGH gene cluster. Biochem. Biophys. Res. Commun. 346, 992-998. doi: 10.1016/j.bbrc.2006.05.199

Blázquez, B., Carmona, M., García, J. L., and Díaz, E. (2008). Identification and analysis of a glutaryl-CoA dehydrogenase-encoding gene and its cognate transcriptional regulator from Azoarcus sp. CIB. Environ. Microbiol. 10, 474-482. doi: 10.1111/j.1462-2920.2007.01468.x

Bozinovski, D., Taubert, M., Kleinsteuber, S., Richnow, H. H., von Bergen, M., Vogt, C., et al. (2014). Metaproteogenomic analysis of a sulfate-reducing enrichment culture reveals genomic organization of key enzymes in the $m$-xylene degradation pathway and metabolic activity of proteobacteria. Syst. Appl. Microbiol. 37, 488-501. doi: 10.1016/j.syapm.2014.07.005

Brow, C. N., O’Brien Johnson, R., Johnson, R. L., and Simon, H. M. (2013). Assessment of anaerobic toluene biodegradation activity by $b s s A$ transcript/gene ratios. Appl. Environ. Microbiol. 79, 5338-5344. doi: 10.1128/ AEM.01031-13

Busch, A., Guazzaroni, M. E., Lacal, J., Ramos, J. L., and Krell, T. (2009). The sensor kinase TodS operates by a multiple step phosphorelay mechanism involving two autokinase domains. J. Biol. Chem. 284, 10353-10360. doi: 10.1074/jbc. M900521200

Busch, A., Lacal, J., Martos, A., Ramos, J. L., and Krell, T. (2007). Bacterial sensor kinase TodS interacts with agonistic and antagonistic signals. Proc. Natl. Acad. Sci. U.S.A. 104, 13774-13779. doi: 10.1073/pnas.0701547104

Busch, A., Lacal, J., Silva-Jímenez, H., Krell, T., and Ramos, J. L. (2010). Catabolite repression of the TodS/TodT two-component system and effectordependent transphosphorylation of TodT as the basis for toluene dioxygenase catabolic pathway control. J. Bacteriol. 192, 4246-4250. doi: 10.1128/JB. 00379-10

Butler, J. E., He, Q., Nevin, K. P., He, Z., Zhou, J., and Lovley, D. R. (2007). Genomic and microarray analysis of aromatics degradation in Geobacter metallireducens and comparison to a Geobacter isolate from a contaminated field site. BMC Genomics 8:180. doi: 10.1186/1471-2164-8-180

Carmona, M., Prieto, M. A., Galán, B., García, J. L., and Díaz, E. (2008). “Signaling networks and design of pollutants biosensors," in Microbial Biodegradation: Genomics and Molecular Biology, ed. E. Díaz (Norfolk, VA: Caister Academic Press), 97-143.

Carmona, M., Zamarro, M. T., Blázquez, B., Durante-Rodríguez, G., Juárez, J. F., Valderrama, J. A., et al. (2009). Anaerobic catabolism of aromatic compounds: a genetic and genomic view. Microbiol. Mol. Biol. Rev. 73, 71-133. doi: 10.1128/ MMBR.00021-08

Cases, I., de Lorenzo, V., and Pérez-Martín, J. (1996). Involvement of sigma 54 in exponential silencing of the Pseudomonas putida TOL plasmid $\mathrm{Pu}$ promoter. Mol. Microbiol. 19, 7-17. doi: 10.1046/j.1365-2958.1996.345873.x

Chakraborty, R., and Coates, J. D. (2004). Anaerobic degradation of monoaromatic hydrocarbons. Appl. Microbiol. Biotechnol. 64, 437-446. doi: 10.1007/s00253003-1526-x

Chaurasia, A. K., Tremblay, P. L., Holmes, D. E., and Zhang, T. (2015). Genetic evidence that the degradation of para-cresol by Geobacter metallireducens is catalyzed by the periplasmic para-cresol methylhydroxylase. FEMS Microbiol. Lett. 362:fnv145. doi: 10.1093/femsle/fnv145

Coschigano, P. W. (2000). Transcriptional analysis of the tutE tutFDGH gene cluster from Thauera aromatica strain T1. Appl. Environ. Microbiol. 66, 1147-1151. doi: 10.1128/AEM.66.3.1147-1151.2000
Coschigano, P. W., and Bishop, B. J. (2004). Role of benzylsuccinate in the induction of the tutE tutFDGH gene complex of T. aromatica strain T1. FEMS Microbiol. Lett. 231, 261-266. doi: 10.1016/S0378-1097(04)00005-9

Coschigano, P. W., and Young, L. Y. (1997). Identification and sequence analysis of two regulatory genes involved in anaerobic toluene metabolism by strain T1. Appl. Environ. Microbiol. 63, 652-660.

de Lorenzo, V., and Timmis, K. N. (1994). Analysis and construction of stable phenotypes in gram-negative bacteria with Tn5- and Tn10-derived minitransposons. Methods Enzymol. 235, 386-405. doi: 10.1016/0076-6879(94) 35157-0

Duetz, W. A., Marqués, S., Wind, B., Ramos, J. L., and van Andel, J. G. (1996). Catabolite repression of the toluene degradation pathway in Pseudomonas putida harboring pWW0 under various conditions of nutrient limitation in chemostat culture. Appl. Environ. Microbiol. 62, 601-606.

Durante-Rodríguez, G., Zamarro, M. T., García, J. L., Díaz, E., and Carmona, M. (2006). Oxygen-dependent regulation of the central pathway for the anaerobic catabolism of aromatic compounds in Azoarcus sp. strain CIB. J. Bacteriol. 188, 2343-2354. doi: 10.1128/JB.188.7.2343-2354.2006

Felsenstein, J. (1993). PHYLIP (Phylogenetic Inference Package) Version 3.5.1. Seattle, WA: University of Washington.

Fernández, H., Prandoni, N., Fernández-Pascual, M., Fajardo, S., Morcillo, C., Díaz, E., et al. (2014). Azoarcus sp. CIB, an anaerobic biodegrader of aromatic compounds shows an endophytic lifestyle. PLoS One 9:e110771. doi: 10.1371/ journal.pone.0110771

Fernández-Llamosas, H., Castro, L., Blázquez, M. L., Díaz, E., and Carmona, M. (2016). Biosynthesis of selenium nanoparticles by Azoarcus sp. CIB. Microb. Cell Fact. 15:109. doi: 10.1186/s12934-016-0510-y

Ferrández, A., Miñambres, B., García, B., Olivera, E. R., Luengo, J. M., García, J. L., et al. (1998). Catabolism of phenylacetic acid in Escherichia coli. Characterization of a new aerobic hybrid pathway. J. Biol. Chem. 273, 25974-25986. doi: 10.1074/jbc.273.40.25974

Foght, J. (2008). Anaerobic biodegradation of aromatic hydrocarbons: pathways and prospects. J. Mol. Microbiol. Biotechnol. 15, 93-120. doi: 10.1159/0001 21324

Funk, M. A., Judd, E. T., Marsh, E. N., Elliott, S. J., and Drennan, C. L. (2014). Structures of benzylsuccinate synthase elucidate roles of accessory subunits in glycyl radical enzyme activation and activity. Proc. Natl. Acad. Sci. U.S.A. 111, 10161-10166. doi: 10.1073/pnas.1405983111

Heider, J., Boll, M., Breese, K., Breinig, S., Ebenau-Jehle, C., Feil, U., et al. (1998). Differential induction of enzymes involved in anaerobic metabolism of aromatic compounds in the denitrifying bacterium Thauera aromatica. Arch. Microbiol. 170, 120-131. doi: 10.1007/s002030050623

Heider, J., Szaleniec, M., Martins, B. M., Seyhan, D., Buckel, W., and Golding, B. T. (2016). Structure and function of benzylsuccinate synthase and related fumarate-adding glycyl radical enzymes. J. Mol. Microbiol. Biotechnol. 26, 29-44. doi: 10.1159/000441656

Hermuth, K., Leuthner, B., and Heider, J. (2002). Operon structure and expression of the genes for benzylsuccinate synthase in Thauera aromatica strain K172. Arch. Microbiol. 177, 132-138. doi: 10.1007/s00203-001-0375-1

Herrmann, S., Vogt, C., Fischer, A., Kuppardt, A., and Richnow, H. H. (2009). Characterization of anaerobic xylene biodegradation by two-dimensional isotope fractionation analysis. Environ. Microbiol. Rep. 1, 535-544. doi: 10.1111/ j.1758-2229.2009.00076.x

Juárez, J. F., Liu, H., Zamarro, M. T., McMahon, S., Liu, H., Naismith, J. H., et al. (2015). Unraveling the specific regulation of the central pathway for anaerobic degradation of 3-methylbenzoate. J. Biol. Chem. 290, 12165-12183. doi: 10.1074/jbc.M115.637074

Juárez, J. F., Zamarro, M. T., Eberlein, C., Boll, M., Carmona, M., and Díaz, E. (2013). Characterization of the mbd cluster encoding the anaerobic 3-methylbenzoyl-CoA central pathway. Environ. Microbiol. 15, 148-166. doi: 10.1111/j.1462-2920.2012.02818.x

Kim, S. J., Park, S. J., Jung, M. Y., Kim, J. G., Madsen, E. L., and Rhee, S. K. (2014). An uncultivated nitrate-reducing member of the genus Herminiimonas degrades toluene. Appl. Environ. Microbiol. 80, 3233-3243. doi: 10.1128/AEM. 03975-13

Kimura, M. (1980). A simple method for estimating evolutionary rates of base substitutions through comparative studies of nucleotide sequences. J. Mol. Evol. 16, 111-120. doi: 10.1007/BF01731581 
Koh, S., Hwang, J., Guchhait, K., Lee, E. G., Kim, S. Y., Kim, S., et al. (2016). Molecular insights into toluene sensing in the TodS/TodT signal transduction system. J. Biol. Chem. 291, 8575-8590. doi: 10.1074/jbc.M116. 718841

Krieger, C. J., Beller, H. R., Reinhard, M., and Spormann, A. M. (1999). Initial reactions in anaerobic oxidation of $m$-xylene by the denitrifying bacterium Azoarcus sp. strain T. J. Bacteriol. 181, 6403-6410.

Krieger, C. J., Roseboom, W., Albracht, S. P., and Spormann, A. M. (2001). A stable organic free radical in anaerobic benzylsuccinate synthase of Azoarcus sp. strain T. J. Biol. Chem. 276, 12924-12927. doi: 10.1074/jbc.M009453200

Kube, M., Heider, J., Amann, J., Hufnagel, P., Kühner, S., Beck, A., et al. (2004). Genes involved in the anaerobic degradation of toluene in a denitrifying bacterium, strain EbN1. Arch. Microbiol. 181, 182-194. doi: 10.1007/s00203003-0627-3

Kühner, S., Wöhlbrand, L., Fritz, I., Wruck, W., Hultschig, C., Hufnagel, P., et al. (2005). Substrate-dependent regulation of anaerobic degradation pathways for toluene and ethylbenzene in a denitrifying bacterium, strain EbN1. J. Bacteriol. 187, 1493-1503. doi: 10.1128/JB.187.4.1493-1503.2005

Kummel, S., Kuntze, K., Vogt, C., Boll, M., Heider, J., and Richnow, H. H. (2013). Evidence for benzylsuccinate synthase subtypes obtained by using stable isotope tools. J. Bacteriol. 195, 4660-4667. doi: 10.1128/JB.00477-13

Lacal, J., Busch, A., Guazzaroni, M. E., Krell, T., and Ramos, J. L. (2006). The TodSTodT two-component regulatory system recognizes a wide range of effectors and works with DNA-bending proteins. Proc. Natl. Acad. Sci. U.S.A. 103, 8191-8196. doi: 10.1073/pnas.0602902103

Leoni, L., Ascenzi, P., Bocedi, A., Rampioni, G., Castellini, L., and Zennaro, E. (2003). Styrene-catabolism regulation in Pseudomonas fluorescens ST: phosphorylation of StyR induces dimerization and cooperative DNA-binding. Biochem. Biophys. Res. Commun. 303, 926-931. doi: 10.1016/S0006-291X(03)00450-9

Leuthner, B., and Heider, J. (1998). A two-component system involved in regulation of anaerobic toluene metabolism in Thauera aromatica. FEMS Microbiol. Lett. 166, 35-41. doi: 10.1111/j.1574-6968.1998.tb13180.x

Leuthner, B., and Heider, J. (2000). Anaerobic toluene catabolism of Thauera aromatica: the $b b$ s operon codes for enzymes of $\beta$ oxidation of the intermediate benzylsuccinate. J. Bacteriol. 182, 272-277. doi: 10.1128/JB.182.2.272277.2000

Leuthner, B., Leutwein, C., Schulz, H., Hörth, P., Haehnel, W., Schiltz, E., et al. (1998). Biochemical and genetic characterization of benzylsuccinate synthase from Thauera aromatica: a new glycyl radical enzyme catalysing the first step in anaerobic toluene metabolism. Mol. Microbiol. 28, 615-628. doi: 10.1046/j. 1365-2958.1998.00826.x

Leutwein, C., and Heider, J. (2001). Succinyl-CoA:(R)-benzylsuccinate CoAtransferase: an enzyme of the anaerobic toluene catabolic pathway in denitrifying bacteria. J. Bacteriol. 183, 4288-4295. doi: 10.1128/JB.183.14.42884295.2001

Leutwein, C., and Heider, J. (2002). (R)-Benzylsuccinyl-CoA dehydrogenase of Thauera aromatica, an enzyme of the anaerobic toluene catabolic pathway. Arch. Microbiol. 178, 517-524. doi: 10.1007/s00203-002-0484-5

Liu, B., Frostegård, A., and Shapleigh, J. P. (2013). Draft genome sequences of five strains in the genus Thauera. Genome Announc. 1:e00052-12. doi: 10.1128/ genomeA.00052-12

López-Barragán, M. J., Carmona, M., Zamarro, M. T., Thiele, B., Boll, M., Fuchs, G., et al. (2004). The bzd gene cluster, coding for anaerobic benzoate catabolism, in Azoarcus sp. strain CIB. J. Bacteriol. 186, 5762-5774. doi: 10.1128/JB.186.17. 5762-5774.2004

Lovley, D. R., Baedecker, M. J., Lonergan, D. J., Cozzarelli, I. M., Phillips, E. J. P., and Siegel, D. I. (1989). Oxidation of aromatic contaminants coupled to microbial iron reduction. Nature 339, 297-300. doi: 10.1038/339297a0

Lueders, T. (2017). The ecology of anaerobic degraders of BTEX hydrocarbons in aquifers. FEMS Microbiol. Ecol. 93:fiw220. doi: 10.1093/femsec/ fiw 220

Lünsmann, V., Kappelmeyer, U., Taubert, A., Nijenhuis, I., von Bergen, M., Heipieper, H. J., et al. (2016). Aerobic toluene degraders in the rhizosphere of a constructed wetland model show diurnal polyhydroxyalkanoate metabolism. Appl. Environ. Microbiol. 82, 4126-4132. doi: 10.1128/AEM.00493-16

Marqués, S., Holtel, A., Timmis, K. N., and Ramos, J. L. (1994). Transcriptional induction kinetics from the promoters of the catabolic pathways of TOL plasmid pWW0 of Pseudomonas putida for metabolism of aromatics. J. Bacteriol. 176, 2517-2524. doi: 10.1128/jb.176.9.2517-2524.1994

Martín-Moldes, Z., Blázquez, B., Baraquet, C., Harwood, C. S., Zamarro, M. T., and Díaz, E. (2016). Degradation of cyclic diguanosine monophosphate by a hybrid two-component protein protects Azoarcus sp. strain CIB from toluene toxicity. Proc. Natl. Acad. Sci. U.S.A. 113, 13174-13179. doi: 10.1073/pnas.1615981113

Martín-Moldes, Z., Zamarro, M. T., del Cerro, C., Valencia, A., Gómez, M. J., Arcas, A., et al. (2015). Whole-genome analysis of Azoarcus sp. strain CIB provides genetic insights to its different lifestyles and predicts novel metabolic features. Syst. Appl. Microbiol. 38, 462-471. doi: 10.1016/j.syapm.2015. 07.002

Meyer-Cifuentes, I., Martinez-Lavanchy, P. M., Marin-Cevada, V., Böhnke, S., Harms, H., Müller, J. A., et al. (2017). Isolation and characterization of Magnetospirillum sp. strain 15-1 as a representative anaerobic toluene-degrader from a constructed wetland model. PLoS One 12:e0174750. doi: 10.1371/ journal.pone.0174750

Milani, M., Leoni, L., Rampioni, G., Zennaro, E., Ascenzi, P., and Bolognesi, M. (2005). An active-like structure in the unphosphorylated StyR response regulator suggests a phosphorylation- dependent allosteric activation mechanism. Structure 13, 1289-1297. doi: 10.1016/j.str.2005.05.014

Miller, J. H. (1972). Experiments in Molecular Genetics. New York, NY: Cold Spring Harbord Laboratory.

Morasch, B., Schink, B., Tebbe, C. C., and Meckenstock, R. U. (2004). Degradation of o-xylene and m-xylene by a novel sulfate-reducer belonging to the genus Desulfotomaculum. Arch. Microbiol. 181, 407-417. doi: 10.1007/s00203-0040672-6

Rabus, R., Boll, M., Heider, J., Meckenstock, R. U., Buckel, W., Einsle, O., et al. (2016). Anaerobic microbial degradation of hydrocarbons: from enzymatic reactions to the environment. J. Mol. Microbiol. Biotechnol. 26, 5-28. doi: 10.1159/000443997

Rabus, R., Kube, M., Beck, A., Widdel, F., and Reinhardt, R. (2002). Genes involved in the anaerobic degradation of ethylbenzene in a denitrifying bacterium, strain EbN1. Arch. Microbiol. 178, 506-516. doi: 10.1007/s00203-002-0487-2

Rojo, F. (2010). Carbon catabolite repression in Pseudomonas: optimizing metabolic versatility and interactions with the environment. FEMS Microbiol. Rev. 34, 658-684. doi: 10.1111/j.1574-6976.2010.00218.x

Ruíz, R., Aranda-Olmedo, M. I., Domínguez-Cuevas, P., Ramos-González, M. I., and Marqués, S. (2004). "Transcriptional regulation of the toluene catabolic pathways," in Pseudomonas Virulence and Gene Regulation, Vol. 2, ed. J. L. Ramos (New York, NY: Kluwer Academic), 509-537.

Saitou, N., and Nei, M. (1987). The neighbor-joining method: a new method for reconstructing phylogenetic trees. Mol. Biol. Evol. 4, 406-425.

Sambrook, J., and Russell, D. W. (2001). Molecular Cloning: A Laboratory Manual. New York, NY: Cold Spring Harbor.

Sanger, F., Nicklen, S., and Coulson, A. R. (1977). DNA sequencing with chainterminating inhibitors. Proc. Natl. Acad. Sci. U.S.A. 74, 5463-5467. doi: 10.1073/ pnas.74.12.5463

Schäfer, A., Tauch, A., Jäger, W., Kalinowski, J., Thierbach, G., and Pühler, A. (1994). Small mobilizable multi-purpose cloning vectors derived from the Escherichia coli plasmids pK18 and pK19: selection of defined deletions in the chromosome of Corynebacterium glutamicum. Gene 145, 69-73. doi: 10.1016/ 0378-1119(94)90324-7

Selmer, T., Pierik, A. J., and Heider, J. (2005). New glycyl radical enzymes catalysing key metabolic steps in anaerobic bacteria. Biol. Chem. 386, 981-988. doi: 10.1515/BC.2005.114

Shinoda, Y., Akagi, J., Uchihashi, Y., Hiraishi, A., Yukawa, H., Yurimoto, H., et al. (2005). Anaerobic degradation of aromatic compounds by Magnetospirillum strains: isolation and degradation genes. Biosci. Biotechnol. Biochem. 69, 1483-1491. doi: 10.1271/bbb.69.1483

Shinoda, Y., Sakai, Y., Uenishi, H., Uchihashi, Y., Hiraishi, A., Yukawa, H., et al. (2004). Aerobic and anaerobic toluene degradation by a newly isolated denitrifying bacterium, Thauera sp. strain DNT-1. Appl. Environ. Microbiol. 70, 1385-1392. doi: 10.1128/AEM.70.3.1385-1392.2004

Silva-Jiménez, H., García-Fontana, C., Cadirci, B. H., Ramos-González, M. I., Ramos, J. L., and Krell, T. (2012). Study of the TmoS/TmoT two-component system: towards the functional characterization of the family of TodS/TodT like systems. Microb. Biotechnol. 5, 489-500. doi: 10.1111/j.1751-7915.2011. 00322.x 
Strijkstra, A., Trautwein, K., Jarling, R., Wöhlbrand, L., Dörries, M., Reinhardt, R., et al. (2014). Anaerobic activation of p-cymene in denitrifying betaproteobacteria: methyl group hydroxylation versus addition to fumarate. Appl. Environ. Microbiol. 80, 7592-7603. doi: 10.1128/AEM.02385-14

Takeda, H., Shimodaira, J., Yukawa, K., Hara, N., Kasai, D., Miyauchi, K., et al. (2010). Dual two-component regulatory systems are involved in aromatic compound degradation in a polychlorinated-biphenyl degrader, Rhodococcus jostii RHA1. J. Bacteriol. 192, 4741-4751. doi: 10.1128/JB. 00429-10

Thompson, J. D., Higgins, D. G., and Gibson, T. J. (1994). CLUSTAL W: improving the sensitivity of progressive multiple sequence alignment through sequence weighting, position-specific gap penalties and weight matrix choice. Nucleic Acids Res. 22, 4673-4680. doi: 10.1093/nar/22.22.4673

Trautwein, K., Kühner, S., Wöhlbrand, L., Halder, T., Kuchta, K., Steinbüchel, A., et al. (2008). Solvent stress response of the denitrifying bacterium "Aromatoleum aromaticum" strain EbN1. Appl. Environ. Microbiol. 74, 2267-2274. doi: 10.1128/AEM.02381-07

Valderrama, J. A., Durante-Rodríguez, G., Blázquez, B., García, J. L., Carmona, M., and Díaz, E. (2012). Bacterial degradation of benzoate: cross-regulation between aerobic and anaerobic pathways. J. Biol. Chem. 287, 10494-10508. doi: 10.1074/ jbc.M111.309005

Valderrama, J. A., Shingler, V., Carmona, M., and Díaz, E. (2014). AccR is a master regulator involved in carbon catabolite repression of the anaerobic catabolism of aromatic compounds in Azoarcus sp. CIB. J. Biol. Chem. 289, 1892-1894. doi: 10.1074/jbc.M113.517714

Velasco, A., Alonso, S., García, J. L., Perera, J., and Díaz, E. (1998). Genetic and functional analysis of the styrene catabolic cluster of Pseudomonas sp. Strain Y2. J. Bacteriol. 180, 1063-1071.
Verfürth, K., Pierik, A. J., Leutwein, C., Zorn, S., and Heider, J. (2004). Substrate specificities and electron paramagnetic resonance properties of benzylsuccinate synthases in anaerobic toluene and m-xylene metabolism. Arch. Microbiol. 181, 155-162. doi: 10.1007/s00203-003-0642-4

Wöhlbrand, L., Jacob, J. H., Kube, M., Mussmann, M., Jarling, R., Beck, A., et al. (2013). Complete genome, catabolic sub-proteomes and key-metabolites of Desulfobacula toluolica Tol2, a marine, aromatic compound-degrading, sulfatereducing bacterium. Environ. Microbiol. 15, 1334-1355. doi: 10.1111/j.14622920.2012.02885.x

Young, L. Y., and Phelps, C. D. (2005). Metabolic biomarkers for monitoring in situ anaerobic hydrocarbon degradation. Environ. Health Perspect. 113, 62-67. doi: 10.1289/ehp.6940

Zamarro, M. T., Martín-Moldes, Z., and Díaz, E. (2016). The ICEXTD of Azoarcus sp. CIB, an integrative and conjugative element with aerobic and anaerobic catabolic properties. Environ. Microbiol. 18, 5018-5031. doi: 10.1111/14622920.13465

Conflict of Interest Statement: The authors declare that the research was conducted in the absence of any commercial or financial relationships that could be construed as a potential conflict of interest.

Copyright (c) 2018 Blázquez, Carmona and Díaz. This is an open-access article distributed under the terms of the Creative Commons Attribution License (CC BY). The use, distribution or reproduction in other forums is permitted, provided the original author(s) and the copyright owner are credited and that the original publication in this journal is cited, in accordance with accepted academic practice. No use, distribution or reproduction is permitted which does not comply with these terms. 